\title{
Theoretical development of elliptic cross-sectional hyperboloidal harmonics and their application to electrostatics
}

Sten, J. C.-E.

2017-05

Sten , J C-E , Fragoyiannis , G , Vafeas , P , Koivisto , P K \& Dassios , G 2017 , ' Theoretical development of elliptic cross-sectional hyperboloidal harmonics and their application to electrostatics ' , Journal of Mathematical Physics , vol. 58 , no. 5 , 053505 . https://doi.org/10.1063/1.4982638

http://hdl.handle.net/10138/299174

https://doi.org/10.1063/1.4982638

unspecified

publishedVersion

Downloaded from Helda, University of Helsinki institutional repository.

This is an electronic reprint of the original article.

This reprint may differ from the original in pagination and typographic detail.

Please cite the original version. 


\section{Theoretical development of elliptic cross-sectional hyperboloidal harmonics and their application to electrostatics}

Cite as: J. Math. Phys. 58, 053505 (2017); https://doi.org/10.1063/1.4982638

Submitted: 28 September 2016 . Accepted: 14 April 2017 . Published Online: 08 May 2017

J. C.-E. Sten, G. Fragoyiannis, P. Vafeas, P. K. Koivisto, and G. Dassios
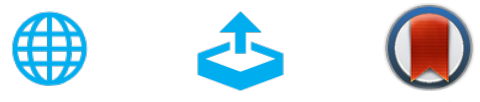

View Online

\section{ARTICLES YOU MAY BE INTERESTED IN}

Phase space distribution of Riemann zeros

Journal of Mathematical Physics 58, 053504 (2017); https://

doi.org/10.1063/1.4982737

On cosmological constant of generalized Robertson-Walker space-times

Journal of Mathematical Physics 58, 053508 (2017); https://

doi.org/10.1063/1.4983134

A fundamental solution to the Schrödinger equation with Doss potentials and its smoothness

Journal of Mathematical Physics 58, 053506 (2017); https://

doi.org/10.1063/1.4983132

\section{Don't let your writing \\ keep you from getting published!}

AIP $\mid$ Author Services

Learn more today! 


\title{
Theoretical development of elliptic cross-sectional hyperboloidal harmonics and their application to electrostatics
}

\author{
J. C.-E. Sten, ${ }^{1}$ G. Fragoyiannis, ${ }^{2}$ P. Vafeas, ${ }^{2, a)}$ P. K. Koivisto, ${ }^{3}$ and \\ G. Dassios ${ }^{2}$ \\ ${ }^{1}$ Department of Mathematics and Statistics, University of Helsinki, P.O. Box 68, \\ FI-00014 Helsinki, Finland \\ ${ }^{2}$ Department of Chemical Engineering, University of Patras, 26504 Patras, Greece \\ ${ }^{3}$ Technical Research Centre of Finland, P.O. Box 1000, FI-02044 Espoo, Finland
}

(Received 28 September 2016; accepted 14 April 2017; published online 8 May 2017)

\begin{abstract}
The analytic computation of electric and magnetic fields near corners and edges is important in many applications related to science and engineering. However, such complicated situations are hard to deal with, since they accumulate charges and consequently they mathematically represent singularities. In order to model this singular behavior, we introduce a novel method, which is related to the geometry and the analysis of the ellipsoidal coordinate system. Indeed, adopting the benefits of the corresponding coordinate surfaces, we use a general non-circular double cone, being the asymptote of a two-sided hyperboloid of two sheets with elliptic cross section, which matches almost perfectly the particular physics and captures the corresponding essential features in a fully three-dimensional fashion. To this end, our analytical technique employs the ellipsoidal geometry and adapts the ellipsoidal functions (solutions of the well-known Lamé equation) so as to construct a new set of the so-called elliptic cross-sectional hyperboloidal harmonics, supplemented by the appropriate orthogonality rules on every constant coordinate surface. By first recollecting the key results of the coordinate system and the related potential functions, including the indispensable orthogonality results, we demonstrate our method to the solution of two boundary value problems in electrostatics. Both refer to a non-penetrable two-hyperboloid of elliptic cross section and its double-cone limit, the first one being charged and the second one scattering off a plane wave. Closed form expressions are derived for the related fields, while the already known formulae from the literature are readily recovered, all cases being followed by the appropriate numerical implementation. Published by AIP Publishing. [http://dx.doi.org/10.1063/1.4982638]
\end{abstract}

\section{INTRODUCTION}

The behavior of electromagnetic fields near corners, edges, and points provides an important knowledge in a number of significant scientific and technical applications. ${ }^{1}$ For instance, when modeling the electrostatic problem around a conductor, the potential is assumed to be constant on the conductor, and the electric field around the conductor is perpendicular to the boundary. However, near a singular point, let us say a corner, the field is perpendicular on all sides leading to an abrupt change of the normal direction. This results to an infinite force on an infinitesimal part of the conductor. Similar problems appear in cases of non-convex edges and points, where in the presence of singularities, the solution fields have no square integrable gradients. However, in practice, there does not exist physical objects that have a perfect corner or edge, since there is always some degree of roundness. In addition, at those locations where the fields become extremely high, other phenomena

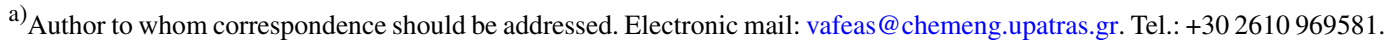


occur simultaneously, which play a crucial role to the limiting values of the field. Hence, the reasons that give this maximum value are not at all trivial.

Most methods to characterize singular fields involve different analytical, variational, and numerical techniques. In particular, the method of separation of variables in combination with the MehlerFock index transform has been used for the study of electromagnetic fields near boundaries having the hyperboloidal shape. ${ }^{2,3}$ Recently, in Ref. 4, two of the authors of the present article applied another straightforward method based on the separation of variables in the spheroidal system of coordinates. Therein, two cases were investigated, the first one where both the two sheets of a perfectly conducting hyperboloid form an electrical boundary and, on the other hand, the case involving only one of the sheets of the hyperboloid, being thus a model of a cone-shaped needle, the apex of which is not perfectly sharp. ${ }^{5}$ Unlike the first problem, the latter one is not confocal for the electric potential because only one of the two sheets of the hyperboloid of circular cross section forms an electrically conducting boundary, on which the potential is constant. In consequence, ordinary spherical harmonics cannot be used in the solution of this problem. Nevertheless, the boundary condition of constant potential will enforce a solution in terms of the order of the related Legendre function, which therefore plays the role of an eigenvalue parameter. In the literature, such special kind of harmonic functions, being of a non-integer order and confined to a circular segment of a sphere, are called "spherical cap-harmonics."

In the present paper, field solutions for non-circular hyperboloids (having in general an elliptic cross section) are investigated by means of the ellipsoidal system of coordinates. ${ }^{6,7}$ The ellipsoidal system of coordinates is the most general coordinate system with second degree coordinate surfaces, in which the Laplace equation is separable. ${ }^{6}$ Consequently, the ellipsoidal shape has been considered in various applications involving electromagnetism, scattering theory, fluid mechanics, geodesy, and other physical subjects (see, e.g., Ref. 6 and the wealth of references therein). However, the ellipsoidal system could as well be called the hyperboloidal system of coordinates, as it is generated by a set of hyperboloids of one and two sheets intersecting each other, as well as the ellipsoidal surfaces orthogonally. To this end, the system of ellipsoidal harmonics and their relation to the hyperboloidal harmonics are first discussed. Because this system is symmetric, it leads to the same Lamé differential equation for each one of three coordinate variables. A similar solution is mutatis mutandis applicable to the hyperboloidal geometry as well. Until now, though, the hyperboloid as a physical model itself has received a very limited attention, see, for example, Refs. 2 and 8. Partly, as in the case of the ellipsoidal coordinate surface of the general two-hyperboloid with an elliptic cross section, this is due to the lack of theoretical preliminaries, a crucial part of which we provide in this article.

There are two kinds of hyperboloids, viz., hyperboloids of one and two sheets. In the following, the attention is focused especially on hyperboloids of two distinct sheets, the so-called two-hyperboloids. Having introduced the hyperboloidal harmonics and demonstrated the orthogonality of them on the surface of a hyperboloid, the theory is next applied to solve basic boundary value problems of electrostatics, such as the problem for the electric potential on a hyperboloid of two sheets. The electric potential generated by a conducting two-hyperboloid having an elliptic cross section, the two sheets oppositely charged, is given analytically in terms of a set of hyperboloidal harmonics composed of Lamé functions of the first kind and given through standard elliptic integrals. ${ }^{9}$ Moreover, in this application, the charge and the capacitance are provided for completeness, while the degenerate case of a two-hyperboloid with circular cross section is obtained and the discussion about how to reach the results for a double cone of either elliptic or circular cross section is being carried out. Also considered is the case of a conducting hyperboloid subject to a transversal low-frequency electromagnetic plane wave field, where both the electric and magnetic problems are presented in terms of the new-defined ellipsoidal-hyperboloidal functions. Contrary to the first application, we provide a brief analysis about the special limit where the two-hyperboloid of a circular cross section is approached, while our attention is mainly focused on the degenerate case where our results are reduced from the twohyperboloidal of elliptic cross-sectional geometry to the double-cone of the circular cross section. Therein, the obtained formulae are given in terms of the associated Legendre functions ${ }^{10}$ and are readily recovered from the initial solution in ellipsoidal coordinates, whereas in accordance with the known results from the literature is readily achieved. Such applications can also be found in Refs. 11 and 12 . 
The paper is organized as follows. In Section II, the geometrical characteristics of the system of ellipsoids and hyperboloids are provided via an analytic mathematical formulation. Some very useful information concerning the geometrical degeneration of the two-hyperboloid of elliptic cross section to the corresponding non-circular double cone, which is of physical interest in the present investigation is also included in Section II. In Section III, the main results of the manuscript, which concern the hyperboloidal harmonics of elliptic cross section and their orthogonality properties are introduced, where the difficulties at each step of the calculations are demonstrated and discussed in details. Moreover, in order to supplement this paper with an analytic application of our generalized method, we invoke Section IV, which is concerned with two basic applications. The first one deals with a perfectly conducting charged two-hyperboloid of non-circular cross section and the second one refers to a non-circular metal two-hyperboloid subject to an incident electric and magnetic plane wave excitation, operating at low frequencies. ${ }^{13}$ The analytical results include plots that depict the variation of the electrostatic potential and electric field between the two sheets of a two-hyperboloid of non-circular cross section and its geometric limits for the two different cases mentioned above. A detailed outline of our work and future steps follows in Section V.

\section{GEOMETRICAL CHARACTERISTICS FOR THE SYSTEM OF ELLIPSOIDS AND HYPERBOLOIDS}

The aforementioned combined system of ellipsoidal-hyperboloidal coordinates is determined by the generating triaxial ellipsoid, depicted in Figure 1(a),

$$
\frac{x_{1}^{2}}{\alpha_{1}^{2}}+\frac{x_{2}^{2}}{\alpha_{2}^{2}}+\frac{x_{3}^{2}}{\alpha_{3}^{2}}=1,
$$

centered at the origin and aligned with the axes of the Cartesian coordinates $\left(x_{1}, x_{2}, x_{3}\right)$. If the semi-principal axes of the reference ellipsoid satisfy $0<\alpha_{3}<\alpha_{2}<\alpha_{1}<+\infty$, then the ellipsoidal coordinates $(\rho, v, \mu)$ of the material point $\mathbf{r} \in \mathbb{R}^{3}$ are connected to the Cartesian ones via the quadratic equations

$$
\begin{gathered}
x_{1}^{2}=\frac{\rho^{2} \mu^{2} v^{2}}{h_{2}^{2} h_{3}^{2}}, \\
x_{2}^{2}=\frac{\left(\rho^{2}-h_{3}^{2}\right)\left(\mu^{2}-h_{3}^{2}\right)\left(h_{3}^{2}-v^{2}\right)}{h_{1}^{2} h_{3}^{2}},
\end{gathered}
$$

and

$$
x_{3}^{2}=\frac{\left(\rho^{2}-h_{2}^{2}\right)\left(h_{2}^{2}-\mu^{2}\right)\left(h_{2}^{2}-v^{2}\right)}{h_{1}^{2} h_{2}^{2}},
$$

(a)

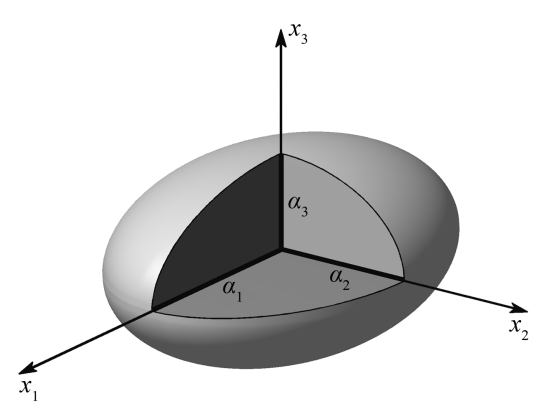

(b)

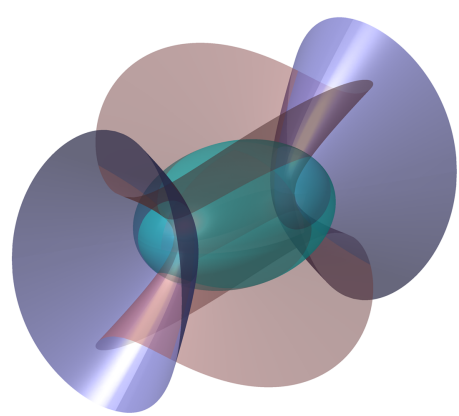

FIG. 1. The ellipsoidal coordinate system, i.e., (a) the reference ellipsoid and (b) the three coordinate surfaces. 
where $h_{1}^{2}=\alpha_{2}^{2}-\alpha_{3}^{2}, h_{2}^{2}=\alpha_{1}^{2}-\alpha_{3}^{2}$, and $h_{3}^{2}=\alpha_{1}^{2}-\alpha_{2}^{2}$ are the semi-focal distances, $h_{2}$ being always larger than $h_{1}$ and $h_{3}$. Since these relationships are quadratic, they do not specify the coordinates of $x_{1}, x_{2}$, and $x_{3}$ uniquely. The variable $\rho$, which plays the role of a "radial" coordinate, varies in the interval $\left[h_{2},+\infty\right)$. On the other hand, for the two "directional" coordinates, there will be adopted the following convention. The variable $\mu \in\left[h_{3}, h_{2}\right]$ is always positive, where the branch of $\sqrt{h_{2}^{2}-\mu^{2}}$ is positive in the direction of growing $\mu$ and negative in the opposite direction. The variable $v$ varies in $\left[-h_{3}, h_{3}\right]$ and backwards, the branch of $\sqrt{h_{3}^{2}-v^{2}}$ being positive in the direction of growing $v$ and negative in the opposite direction. However, in the first octant, whereas all variables $x_{1}, x_{2}$, and $x_{3}$ are positive, the interval of variations of $v$ is $\left[0, h_{3}\right]$, though, in this work, we keep the initial convention, being convenient for our applications.

The quadric surface formed by the variation of the coordinate variables $\mu$ and $\nu$, keeping $\rho$ as a constant, is the ellipsoid

$$
\frac{x_{1}^{2}}{\rho^{2}}+\frac{x_{2}^{2}}{\rho^{2}-h_{3}^{2}}+\frac{x_{3}^{2}}{\rho^{2}-h_{2}^{2}}=1 \text { for every } \rho^{2} \in\left(h_{2}^{2},+\infty\right),
$$

the surface corresponding to the variation of $\rho$ and $v$, keeping $\mu$ constant, is a hyperboloid of one sheet, the so-called one-hyperboloid, given by

$$
\frac{x_{1}^{2}}{\mu^{2}}+\frac{x_{2}^{2}}{\mu^{2}-h_{3}^{2}}-\frac{x_{3}^{2}}{h_{2}^{2}-\mu^{2}}=1 \text { for every } \mu^{2} \in\left(h_{3}^{2}, h_{2}^{2}\right),
$$

and that corresponding to the variation of $\rho$ and $\mu$, keeping $v$ constant, which is a hyperboloid of two sheets, the so-called two-hyperboloid, given via

$$
\frac{x_{1}^{2}}{v^{2}}-\frac{x_{2}^{2}}{h_{3}^{2}-v^{2}}-\frac{x_{3}^{2}}{h_{2}^{2}-v^{2}}=1 \text { for every } v^{2} \in\left(0, h_{3}^{2}\right),
$$

the above coordinate surfaces being sketched in Figure 1(b). The values $\rho=h_{2}$ and $\mu=h_{3}$ specify the focal ellipse and the focal hyperbola, respectively.

Due to our physical implementation described earlier, our main interest is the two-hyperboloid, whose asymptote for large values of $x_{1}, x_{2}$, and $x_{3}$ is approximated by the cone

$$
\frac{x_{2}^{2}}{h_{3}^{2}-v^{2}}+\frac{x_{3}^{2}}{h_{2}^{2}-v^{2}}=\frac{x_{1}^{2}}{v^{2}} \text { for every } v^{2} \in\left(0, h_{3}^{2}\right),
$$

where $x_{1}$ is the central axis of symmetry, while any intersection by a plane parallel to the $x_{1}=0$ plane is an ellipse. The angle, which the positive $v$ branch of the cone forms with the $x_{1}$-axis, is given by the formula

$$
\alpha=\tan ^{-1}\left(\frac{\sqrt{h_{3}^{2}-v^{2}}}{v}\right)=\cos ^{-1}\left(\frac{v}{h_{3}}\right) \text { for any } v \in\left[-h_{3}, h_{3}\right],
$$

in the $x_{3}=0$ plane and

$$
\beta=\tan ^{-1}\left(\frac{\sqrt{h_{2}^{2}-v^{2}}}{v}\right)=\cos ^{-1}\left(\frac{v}{h_{2}}\right) \text { for any } v \in\left[-h_{3}, h_{3}\right],
$$

in the $x_{2}=0$ plane. Thus, the aspect ratio of the cone is

$$
\varepsilon=\frac{\sqrt{h_{3}^{2}-v^{2}}}{\sqrt{h_{2}^{2}-v^{2}}} \text { with } v^{2} \in\left[0, h_{3}^{2}\right],
$$

which coincides with the aspect ratio of the two-hyperboloid from which the cone has been asymptotically reclaimed. 
In terms of the metric coefficients of the ellipsoidal-hyperboloidal coordinate system

$$
h_{\rho}=\frac{\sqrt{\rho^{2}-\mu^{2}} \sqrt{\rho^{2}-v^{2}}}{\sqrt{\rho^{2}-h_{3}^{2}} \sqrt{\rho^{2}-h_{2}^{2}}}, h_{\mu}=\frac{\sqrt{\rho^{2}-\mu^{2}} \sqrt{\mu^{2}-v^{2}}}{\sqrt{\mu^{2}-h_{3}^{2}} \sqrt{h_{2}^{2}-\mu^{2}}}, \text { and } h_{v}=\frac{\sqrt{\rho^{2}-v^{2}} \sqrt{\mu^{2}-v^{2}}}{\sqrt{h_{3}^{2}-v^{2}} \sqrt{h_{2}^{2}-v^{2}}},
$$

as well as taking under our consideration the Jacobian determinant $J=h_{\rho} h_{\mu} h_{v}$ for every $\rho \in\left(h_{2},+\infty\right)$, $\mu \in\left(h_{3}, h_{2}\right)$, and $v \in\left(-h_{3}, h_{3}\right)$, the gradient and the Laplace's differential operators in the ellipsoidal geometry

$$
\nabla=\frac{\hat{\boldsymbol{\rho}}}{h_{\rho}} \frac{\partial}{\partial \rho}+\frac{\hat{\boldsymbol{\mu}}}{h_{\mu}} \frac{\partial}{\partial \mu}+\frac{\hat{\boldsymbol{v}}}{h_{\nu}} \frac{\partial}{\partial v}
$$

and

$$
\Delta=\frac{1}{J}\left\{\frac{\partial}{\partial \rho}\left[\frac{J}{h_{\rho}^{2}} \frac{\partial}{\partial \rho}\right]+\frac{\partial}{\partial \mu}\left[\frac{J}{h_{\mu}^{2}} \frac{\partial}{\partial \mu}\right]+\frac{\partial}{\partial v}\left[\frac{J}{h_{\nu}^{2}} \frac{\partial}{\partial v}\right]\right\},
$$

respectively, are written via the orthonormal coordinate vectors of the system. The outward unit normal vectors on each of the three coordinate surfaces (5)-(7) assume the form

$$
\hat{\boldsymbol{\rho}}=\frac{\rho}{h_{\rho}} \sum_{j=1}^{3} \frac{x_{j} \hat{\boldsymbol{x}}_{j}}{\rho^{2}-\alpha_{1}^{2}+\alpha_{j}^{2}}, \hat{\boldsymbol{\mu}}=\frac{\mu}{h_{\mu}} \sum_{j=1}^{3} \frac{x_{j} \hat{\boldsymbol{x}}_{j}}{\mu^{2}-\alpha_{1}^{2}+\alpha_{j}^{2}}, \text { and } \hat{\boldsymbol{v}}=\frac{v}{h_{\nu}} \sum_{j=1}^{3} \frac{x_{j} \hat{\boldsymbol{x}}_{j}}{v^{2}-\alpha_{1}^{2}+\alpha_{j}^{2}} .
$$

On the other hand, the unit dyadic tensor in the ellipsoidal coordinate system is written as $\tilde{\boldsymbol{I}}=\hat{\boldsymbol{\rho}} \otimes \hat{\boldsymbol{\rho}}+\hat{\boldsymbol{\mu}} \otimes \hat{\boldsymbol{\mu}}+\hat{\boldsymbol{v}} \otimes \hat{\boldsymbol{v}}$.

The strict inequalities $0<\alpha_{3}<\alpha_{2}<\alpha_{1}<+\infty$ form the basic reason why the triaxial ellipsoid reflects the general anisotropy of the three dimensional space. As it is well known, the reduction of general results from the ellipsoidal to the spheroidal, or to the spherical geometry, is not straightforward, since certain indeterminacies appear during the limiting process. This is due to the fact that the spherical system springs from a zero dimensional manifold, i.e., the center, while the ellipsoidal system springs from a two dimensional manifold, i.e., the focal ellipse. The equality of any of the two axes degenerates the ellipsoid to a spheroid, whose axial symmetry is dictated by the third axis. A prolate spheroid is obtained whenever $0<\alpha_{3}=\alpha_{2}<\alpha_{1}<+\infty$ (with the semifocal distances taken as $h_{1}=0$ and $h_{2}=h_{3}=c>0$ ), while the case of an oblate spheroidal shape corresponds to $0<\alpha_{3}<\alpha_{2}$ $=\alpha_{1}<+\infty$ (with the semifocal distances taken as $h_{3}=0$ and $h_{1}=h_{2}=\bar{c}>0$ ). The axis of symmetry is the $x_{1}$-axis for the prolate spheroid and the $x_{3}$-axis for the oblate spheroid. The asymptotic case of the needle can be reached by a prolate spheroid where $0<\alpha_{3}=\alpha_{2} \ll \alpha_{1}<+\infty$, whilst in the case where $0<\alpha_{3} \ll \alpha_{2}=\alpha_{1}<+\infty$, the oblate spheroid takes the shape of a circular disk. The simple transformation $c \rightarrow-i \bar{c}$ allows the transition from the prolate to the oblate spheroid, while the replacement $\bar{c} \rightarrow i c$ leads to the converse. On the other hand, the sphere corresponds to $\alpha_{1}=\alpha_{2}=\alpha_{3}$ $=a$, where $a>0$ is the radius, while in this case, $h_{\kappa}=0$ for $\kappa=1,2,3$. Obviously, similar reduction rules are being followed by the three coordinate surfaces of the ellipsoidal system (5)-(7) for every kind of degenerated shape.

\section{HYPERBOLOIDAL HARMONICS OF ELLIPTIC CROSS SECTION AND ORTHOGONALITY PROPERTIES}

In order to expand a scalar harmonic potential function $u$, which belongs to the kernel space of the Laplace's operator (14), i.e.,

$$
\Delta u(\rho, \mu, v)=0 \text { for } \rho \in\left[h_{2},+\infty\right), \mu \in\left[h_{3}, h_{2}\right] \text {, and } v \in\left[-h_{3}, h_{3}\right] \text {, }
$$

in general or within any prescribed domain of physical interest, we need to construct the appropriate harmonic eigenfunctions valid on both sides of the three coordinate surfaces (5)-(7) of the ellipsoidal system, distinguished by the corresponding constant-variable surfaces. Those will provide us with the corresponding complete eigensolutions satisfying Laplace's equation (16). This procedure leads to the Lamé's equation

$$
\left(x^{2}-h_{3}^{2}\right)\left(x^{2}-h_{2}^{2}\right) E^{\prime \prime}(x)+x\left(2 x^{2}-h_{3}^{2}-h_{2}^{2}\right) E^{\prime}(x)+\left[B-n(n+1) x^{2}\right] E(x)=0 \text { with } n \geq 0,
$$


for each variable $x \equiv \rho, \mu, v$, where prime denotes the derivation with respect to the argument and $B$ is the second separation constant to be determined.

Solutions of (17) are the Lamé functions of the first kind $E=E_{n}(x)$ for $n \geq 0$, whereas a detailed analysis of Equation (17) leads to closed form solutions, which belong to four different classes known as Lamé classes K, L, M, and N (see Ref. 6). The solutions that belong to each class are being structured as follows:

$$
\begin{gathered}
\text { Class } \mathrm{K} / \frac{n}{2}+1(n \text { : even }) \text { and } \frac{n+1}{2}(n \text { : odd }): \quad E_{n}^{K}(x)=\Pi_{n}(x), n \geq 0, \\
\text { Class } \mathrm{L} / \frac{n}{2}(n \text { : even }) \text { and } \frac{n+1}{2}(n \text { : odd }): \quad E_{n}^{L}(x)=\sqrt{\left|x^{2}-h_{3}^{2}\right|} \Pi_{n-1}(x), n \geq 1, \\
\text { Class } \mathrm{M} / \frac{n}{2}(n \text { : even }) \text { and } \frac{n+1}{2}(n \text { : odd }): \quad E_{n}^{M}(x)=\sqrt{\left|x^{2}-h_{2}^{2}\right|} \Pi_{n-1}(x), n \geq 1
\end{gathered}
$$

and

$$
\text { Class N } / \frac{n}{2}(n \text { : even }) \text { and } \frac{n-1}{2}(n: \text { odd }): \quad E_{n}^{N}(x)=\sqrt{\left|x^{2}-h_{3}^{2}\right|} \sqrt{\left|x^{2}-h_{2}^{2}\right|} \Pi_{n-2}(x), n \geq 2,
$$

providing a total of $2 n+1$ independent solutions for every $n \geq 0$ (even or odd), where

$$
\Pi_{n}(x)=\sum_{k=0}^{\left[\frac{n}{2}\right]} a_{k} x^{n-2 k} \text { for } n \in \mathrm{N}
$$

is a polynomial in $x=\rho, \mu, v$, where the $\frac{n}{2}+1$ (if $n$ is even) and the $\frac{n+1}{2}$ (if $n$ is odd) constant coefficients are determined so that each function of (18)-(21) satisfies the Lamé equation (17). These functions are adequate to provide us with the fundamental tools for developing the appropriate theory for the elliptic cross-sectional hyperboloid harmonics. We denote the Lamé functions of degree $n=0,1, \ldots$ and order $m=1,2, \ldots, 2 n+1$ by $E_{n}^{m}=E_{n}^{m}(x)$ for $x=\rho, \mu, v$, which are not bounded at infinity with the exception of $E_{0}^{1}=1$, being regular everywhere, yielding the so-called solutions of the first kind. Aiming to demonstrate this notation, we give an example of the original class of each $E_{n}^{m}$ for $n \leq 3$ (degree of well-known closed formulae ${ }^{6}$ ) and $m=1,2, \ldots, 2 n+1$. Up to that degree, class $\mathrm{K}$ contains six functions, i.e., $E_{0}^{1}, E_{1}^{1}, E_{2}^{1}, E_{2}^{2}, E_{3}^{1}$, and $E_{3}^{2}$, classes $\mathrm{L}$ and $\mathrm{M}$ are comprised of the four functions $E_{1}^{2}, E_{2}^{3}, E_{3}^{3}, E_{3}^{4}$ and $E_{1}^{3}, E_{2}^{4}, E_{3}^{5}, E_{3}^{6}$, respectively, while class $\mathrm{N}$ provides two more functions, $E_{2}^{5}$ and $E_{3}^{7}$, in sum, $6+4+4+2=16$ Lamé functions. It is also known ${ }^{6}$ that all the roots of the Lamé functions are real, unequal, and belong to the interval $\left[-h_{2}, h_{2}\right]$, where the polynomial part of (18)-(21) does not vanish for $x= \pm h_{3}$ and $x= \pm h_{2}$. The second set of linearly independent functions, regular at infinity, is given by

$$
F_{n}^{m}(x)=E_{n}^{m}(x) \int_{x_{c}}^{x} \frac{d t}{\left[E_{n}^{m}(t)\right]^{2} \sqrt{\left|t^{2}-h_{2}^{2}\right|} \sqrt{\left|t^{2}-h_{3}^{2}\right|}} \text { for every } n \geq 0 \text { and } m=1,2, \ldots, 2 n+1,
$$

which stand for the corresponding solutions of the second kind. Herein, $x_{c}$ is appropriately chosen depending on the kind of the boundary or limiting conditions enforced by the corresponding physical problem, whilst the absolute values within the dominator of the expression of integrand (23) are handled accordingly.

We aim at constructing effective mathematical tools for solving physical problems, using solutions of Laplace's equation (16) and involving ellipsoidal boundaries. To this end, the Lamé products,

$$
\mathrm{E}_{n, e}^{m}(\rho, \mu, v)=E_{n}^{m}(\rho) S_{n, e}^{m}(\mu, v) \text { with } \rho \in\left[h_{2},+\infty\right) \text { and for any } \mu \in\left[h_{3}, h_{2}\right], v \in\left[-h_{3}, h_{3}\right],
$$

define the solid ellipsoidal harmonics of the first kind, while the rest involved products,

$$
\mathrm{F}_{n, e}^{m}(\rho, \mu, v)=F_{n}^{m}(\rho) S_{n, e}^{m}(\mu, v) \text { with } \rho \in\left[h_{2},+\infty\right) \text { and for any } \mu \in\left[h_{3}, h_{2}\right], v \in\left[-h_{3}, h_{3}\right],
$$

denote the solid ellipsoidal harmonics of the second kind. Definitions (24) and (25) include the prementioned Lamé functions of each one of the classes (18)-(21) with (22) and for every value of 
$n \geq 0$ and $m=1,2, \ldots, 2 n+1$. Special care should be taken as $\rho \rightarrow+\infty$, depending on the problem under consideration, since $\mathrm{E}_{n, e}^{m}$, except $\mathrm{E}_{0, e}^{1}$, become infinite, while $\mathrm{F}_{n, e}^{m}$ remain bounded at infinity. On the other hand, for $\rho=h_{2}$, both (24) and (25) are applicable as a result of the regular behavior of $\mathrm{F}_{n, e}^{m}$ via a standard limiting process of $F_{n}^{m}(\rho)$, given by (23), as $\rho \rightarrow h_{2}$. Consequently, any function $u_{e}$ that satisfies (16) admits the expansion

$$
u_{e}(\rho, \mu, v)=\sum_{n=0}^{\infty} \sum_{m=1}^{2 n+1}\left[A_{n, e}^{m} \mathrm{E}_{n, e}^{m}(\rho, \mu, v)+B_{n, e}^{m} \mathrm{~F}_{n, e}^{m}(\rho, \mu, v)\right],
$$

for $\rho \in\left[h_{2},+\infty\right), \mu \in\left[h_{3}, h_{2}\right]$, and $v \in\left[=h_{3}, h_{3}\right]$, where $A_{n, e}^{m}$ and $B_{n, e}^{m}$ for $n \geq 0$ and $m=1,2, \ldots, 2 n$ +1 are the unknown constant coefficients to be fixed by the auxiliary conditions of each specific problem, including vanishing of the non-regular parts as the case might be.

Now, let us consider a fixed ellipsoidal surface for $\rho=\rho_{s} \in\left(h_{2},+\infty\right)$. The corresponding complete orthogonal set,

$$
S_{n, e}^{m}(\mu, v)=E_{n}^{m}(\mu) E_{n}^{m}(\nu) \text { for every } \mu \in\left[h_{3}, h_{2}\right] \text { and } v \in\left[-h_{3}, h_{3}\right],
$$

forms the surface ellipsoidal harmonics on the ellipsoid $\rho=\rho_{s}$, enjoying the orthogonality relation

$$
\iint_{\rho=\rho_{s}} S_{n, e}^{m}(\mu, v) S_{n^{\prime}, e}^{m^{\prime}}(\mu, v) \frac{d s_{e}(\mu, v)}{\sqrt{\left(\rho_{s}^{2}-\mu^{2}\right)\left(\rho_{s}^{2}-v^{2}\right)}}=\gamma_{n, e}^{m} \delta_{n n^{\prime}} \delta_{m m^{\prime}} \text { with } d s_{e}(\mu, v)=h_{\mu} h_{v} d \mu d v,
$$

for $n \geq 0, m=1,2, \ldots, 2 n+1$ and $n^{\prime} \geq 0, m^{\prime}=1,2, \ldots, 2 n^{\prime}+1$, where the $\delta$-symbol is the kronecker delta, while the ellipsoidal normalization constants read as

$$
\gamma_{n, e}^{m}=\iint_{\rho=\rho_{s}}\left[S_{n, e}^{m}(\mu, v)\right]^{2} \frac{d s_{e}(\mu, v)}{\sqrt{\left(\rho_{s}^{2}-\mu^{2}\right)\left(\rho_{s}^{2}-v^{2}\right)}} \text { for } n \geq 0 \text { and } m=1,2, \ldots, 2 n+1,
$$

the integration range extending over $\mu \in\left[h_{3}, h_{2}\right]$ and $v \in\left[-h_{3}, h_{3}\right]$.

Similarly, taking under consideration the remaining coordinate surfaces and wishing to deal with boundary value problems involving hyperboloids of elliptic cross section, we proceed to the one-hyperboloid case. Seeking for the appropriate regular harmonic solutions for this situation, we utilize definitions (18)-(23), observing that the eigenfunctions,

$$
\mathrm{E}_{n, 1 h}^{m}(\rho, \mu, v)=E_{n}^{m}(\mu) S_{n, 1 h}^{m}(\rho, v) \text { with } \mu \in\left[h_{3}, h_{2}\right] \text { and for any } \rho \in\left[h_{2},+\infty\right), v \in\left[-h_{3}, h_{3}\right],
$$

represent the solid one-hyperboloidal harmonics of the first kind, whilst

$$
\mathrm{F}_{n, 1 h}^{m}(\rho, \mu, v)=F_{n}^{m}(\mu) S_{n, 1 h}^{m}(\rho, v) \text { with } \mu \in\left[h_{3}, h_{2}\right] \text { and for any } \rho \in\left[h_{2},+\infty\right), v \in\left[-h_{3}, h_{3}\right]
$$

stand for the solid one-hyperboloidal harmonics of the second kind, both yielding bounded expressions for the entire range of the family of hyperboloids of one sheet. Herein, eigensolutions (30) and (31) include the Lamé functions of all classes K, L, M, and N in (18)-(21), given (22) and for every value of $n \geq 0$ and $m=1,2, \ldots, 2 n+1$. Consequently, any harmonic potential $u_{1 h}$, which is the solution of (16) for a general one-hyperboloid, may be expressed in the same basic form as previously via

$$
u_{1 h}(\rho, \mu, v)=\sum_{n=0}^{\infty} \sum_{m=1}^{2 n+1}\left[A_{n, 1 h}^{m} \mathrm{E}_{n, 1 h}^{m}(\rho, \mu, v)+B_{n, 1 h}^{m} \mathrm{~F}_{n, 1 h}^{m}(\rho, \mu, v)\right],
$$

for any $\rho \in\left[h_{2},+\infty\right), \mu \in\left[h_{3}, h_{2}\right]$, and $v \in\left[-h_{3}, h_{3}\right]$, where $A_{n, 1 h}^{m}$ and $B_{n, 1 h}^{m}$ for $n \geq 0$ and $m=1,2, \ldots, 2 n$ +1 denote the undetermined constant coefficients that can be readily evaluated upon the introduction of a specific boundary values, eliminating the unnecessary parts of the expansion accordingly.

Again, we assume a prescribed point at $\mu=\mu_{s} \in\left(h_{3}, h_{2}\right)$, which specifies the particular surface of the hyperboloid of one sheet. Then, as far as the orthogonality property of the corresponding surface harmonics,

$$
S_{n, 1 h}^{m}(\rho, v)=E_{n}^{m}(\rho) E_{n}^{m}(v) \text { for every } \rho \in\left[h_{2},+\infty\right) \text { and } v \in\left[-h_{3}, h_{3}\right],
$$

is concerned, we observe that the only difficulty here is that the surface area of the one-hyperboloid at $\mu=\mu_{s}$ is unbounded, unlike that of the ellipsoid, which is always bounded, implying that the relevant 
surface integral is an improper integral. However, knowing that for large arguments $\rho$, the leading behavior of $E_{n}^{m}(\rho) \rightarrow \rho^{n}, n \geq 0$ for all $m=1,2, \ldots, 2 n+1$, it is easy to see that the following limit gives:

$\lim _{P \rightarrow+\infty} \frac{2 n+1}{P^{2 n+1}} \int_{h_{2}}^{P} \frac{E_{n}^{m}(\rho) E_{n^{\prime}}^{m^{\prime}}(\rho)\left(\rho^{2}-v^{2}\right)}{\sqrt{\left(\rho^{2}-h_{3}^{2}\right)\left(\rho^{2}-h_{2}^{2}\right)}} d \rho=1$ for $v \in\left[-h_{3}, h_{3}\right]$ with $n \geq 0$ and $m=1,2, \ldots, 2 n+1$,

when $n^{\prime}=n=0,1,2, \ldots$ (note $\left.m^{\prime}=1,2, \ldots, 2 n^{\prime}+1\right)$ and otherwise zero. In addition to that, the definite integral

$$
\int_{-h_{3}}^{h_{3}} \frac{E_{n}^{m}(v) E_{n}^{m^{\prime}}(v)}{\sqrt{\left(h_{3}^{2}-v^{2}\right)\left(h_{2}^{2}-v^{2}\right)}} d v=0 \text { for } n \geq 0 \text { and } m, m^{\prime}=1,2, \ldots, 2 n+1,
$$

unless $m=m^{\prime}$. The integral now extends over the entire one-hyperboloid, i.e., first $v$ runs from $-h_{3}$ to $h_{3}$ along the positive branch of $\sqrt{h_{3}^{2}-v^{2}}$ and then back from $h_{3}$ to $-h_{3}$ along the negative branch of the same square root. This property uses the orthogonality among the Lamé functions of the same degree $n \geq 0$ and order $m=1,2, \ldots, 2 n+1$, as well as the different parity of the Lamé functions of the same degree but different order. Hence, we readily conclude that for the one-hyperboloid, the value of the orthogonality expression is

$$
\lim _{P \rightarrow+\infty} \frac{2 n+1}{P^{2 n+1}} \int_{-h_{3}}^{h_{3}} \int_{h_{2}}^{P} \frac{S_{n, 1 h}^{m}(\rho, v) S_{n^{\prime}, 1 h}^{m^{\prime}}(\rho, v)\left(\rho^{2}-v^{2}\right)}{\sqrt{\left(\rho^{2}-h_{3}^{2}\right)\left(\rho^{2}-h_{2}^{2}\right)\left(h_{3}^{2}-v^{2}\right)\left(h_{2}^{2}-v^{2}\right)}} d \rho d v=\gamma_{n, 1 h}^{m} \delta_{n n^{\prime}} \delta_{m m^{\prime}}
$$

or

$$
\lim _{P \rightarrow+\infty} \frac{2 n+1}{P^{2 n+1}} \int_{-h_{3}}^{h_{3}} \int_{h_{2}}^{P} S_{n, 1 h}^{m}(\rho, v) S_{n^{\prime}, 1 h}^{m^{\prime}}(\rho, v) \frac{d s_{1 h}(\rho, v)}{\sqrt{\left(\rho^{2}-\mu_{s}^{2}\right)\left(\mu_{s}^{2}-v^{2}\right)}}=\gamma_{n, 1 h}^{m} \delta_{n n^{\prime}} \delta_{m m^{\prime}}
$$

with $d s_{1 h}(\rho, v)=h_{\rho} h_{v} d \rho d v$, for every $n \geq 0, m=1,2, \ldots, 2 n+1$ and $n^{\prime} \geq 0, m^{\prime}=1,2, \ldots, 2 n^{\prime}$ +1 , which admits a similar expression to (28). Of course, the new one-hyperboloidal constants of orthonormalization are

$$
\gamma_{n, 1 h}^{m}=\lim _{P \rightarrow+\infty} \frac{2 n+1}{P^{2 n+1}} \int_{-h_{3}}^{h_{3}} \int_{h_{2}}^{P}\left[S_{n, 1 h}^{m}(\rho, v)\right]^{2} \frac{d s_{1 h}(\rho, v)}{\sqrt{\left(\rho^{2}-\mu_{s}^{2}\right)\left(\mu_{s}^{2}-v^{2}\right)}} \text { for } n \geq 0 \text { and } m=1,2, \ldots, 2 n+1,
$$

providing all the necessary information regarding the orthogonality properties on the surface of any constant one-hyperboloid $\mu=\mu_{s}$.

Things do not differ much when we are dealing with problems, where hyperboloids of two sheets can be used to model the corresponding physics. As already mentioned, many interesting applications, e.g., in electrostatics, involve proper potential-field evaluations at edges or corners, where their complicated geometrical characteristics can be represented by a double-cone of either circular or, more general, ellipsoidal cross section, which is the limit of the corresponding twohyperboloid. Such cases demand analytic techniques to obtain the final solution in a closed form. Therefore, it is certainly necessary to introduce the implicated eigenfunctions to this case, properly chosen so as to be regular within the interval where the family of hyperboloids of two sheets is generated. Some trivial analysis based on the developed theory in between (18) and (23), concerning the Lamé functions of every class $\mathrm{K}, \mathrm{L}, \mathrm{M}$, and N, leads to the so-called solid two-hyperboloidal harmonics of the first kind

$$
\mathrm{E}_{n, 2 h}^{m}(\rho, \mu, v)=E_{n}^{m}(v) S_{n, 2 h}^{m}(\rho, \mu) \text { with } v \in\left[-h_{3}, h_{3}\right] \text { and for any } \rho \in\left[h_{2},+\infty\right), \mu \in\left[h_{3}, h_{2}\right],
$$


while the complementary solid two-hyperboloidal harmonics of the second kind imply

$$
\mathrm{F}_{n, 2 h}^{m}(\rho, \mu, v)=F_{n}^{m}(v) S_{n, 2 h}^{m}(\rho, \mu) \text { with } v \in\left[-h_{3}, h_{3}\right] \text { and for any } \rho \in\left[h_{2},+\infty\right), \mu \in\left[h_{3}, h_{2}\right] .
$$

Herein, eigensolutions (39) and (40) include the Lamé functions of all classes (18)-(21) with (22) for $n \geq 0$ and $m=1,2, \ldots, 2 n+1$. The potential function $u_{2 h}$, which belongs to the kernel of the Laplace's operator (standard general solution of (16)) is written as

$$
u_{2 h}(\rho, \mu, v)=\sum_{n=0}^{\infty} \sum_{m=1}^{2 n+1}\left[A_{n, 2 h}^{m} \mathrm{E}_{n, 2 h}^{m}(\rho, \mu, v)+B_{n, 2 h}^{m} \mathrm{~F}_{n, 2 h}^{m}(\rho, \mu, v)\right],
$$

for every $\rho \in\left[h_{2},+\infty\right), \mu \in\left[h_{3}, h_{2}\right]$, and $v \in\left[-h_{3}, h_{3}\right]$. The constant coefficients $A_{n, 2 h}^{m}$ and $B_{n, 2 h}^{m}$ for $n \geq 0$ and $m=1,2, \ldots, 2 n+1$ must be determined with respect to a well-defined physical problem, wherein the unnecessary terms correspond to zero values.

With respect to a fixed two-hyperboloid for $v=-v_{s} \in\left[-h_{3}, 0\right)$ or $v=v_{s} \in\left(0, h_{3}\right]$, an orthogonality relation for the surface two-hyperboloidal harmonics,

$$
S_{n, 2 h}^{m}(\rho, \mu)=E_{n}^{m}(\rho) E_{n}^{m}(\mu) \text { for every } \rho \in\left[h_{2},+\infty\right) \text { and } \mu \in\left[h_{3}, h_{2}\right],
$$

valid on the surface of a two-hyperboloid $v= \pm v_{s}$, reads as

$$
\lim _{P \rightarrow+\infty} \frac{2 n+1}{P^{2 n+1}} \int_{h_{3}}^{h_{2}} \int_{h_{2}}^{P} \frac{S_{n, 2 h}^{m}(\rho, \mu) S_{n^{\prime}, 2 h}^{m^{\prime}}(\rho, \mu)\left(\rho^{2}-\mu^{2}\right)}{\sqrt{\left(\rho^{2}-h_{3}^{2}\right)\left(\rho^{2}-h_{2}^{2}\right)\left(\mu^{2}-h_{3}^{2}\right)\left(h_{2}^{2}-\mu^{2}\right)}} d \rho d \mu=\gamma_{n, 2 h}^{m} \delta_{n n^{\prime}} \delta_{m m^{\prime}}
$$

or

$$
\lim _{P \rightarrow+\infty} \frac{2 n+1}{P^{2 n+1}} \int_{h_{3}}^{h_{2}} \int_{h_{2}}^{P} S_{n, 2 h}^{m}(\rho, \mu) S_{n^{\prime}, 2 h}^{m^{\prime}}(\rho, \mu) \frac{d s_{2 h}(\rho, \mu)}{\sqrt{\left(\rho^{2}-v_{s}^{2}\right)\left(\mu^{2}-v_{s}^{2}\right)}}=\gamma_{n, 2 h}^{m} \delta_{n n^{\prime}} \delta_{m m^{\prime}}
$$

with $d s_{2 h}(\rho, \mu)=h_{\rho} h_{\mu} d \rho d \mu$, for every $n \geq 0, m=1,2, \ldots, 2 n+1$ and $n^{\prime} \geq 0, m^{\prime}=1,2, \ldots, 2 n^{\prime}+1$. The relevant two-hyperboloidal constants of orthonormalization are

$$
\gamma_{n, 2 h}^{m}=\lim _{P \rightarrow+\infty} \frac{2 n+1}{P^{2 n+1}} \int_{h_{3}}^{h_{2}} \int_{h_{2}}^{P}\left[S_{n, 2 h}^{m}(\rho, \mu)\right]^{2} \frac{d s_{2 h}(\rho, \mu)}{\sqrt{\left(\rho^{2}-v_{s}^{2}\right)\left(\mu^{2}-v_{s}^{2}\right)}} \text { for } n \geq 0 \text { and } m=1,2, \ldots, 2 n+1 .
$$

Validity of relation (43) rests on similar arguments as given previously in connection to (34), i.e., the parity of Lamé functions of the same degree $n \geq 0$ but different order $m=1,2, \ldots, 2 n+1$, as well as the integral property

$$
\int_{h_{3}}^{h_{2}} \frac{E_{n}^{m}(\mu) E_{n}^{m^{\prime}}(\mu)}{\sqrt{\left(\mu^{2}-h_{3}^{2}\right)\left(h_{2}^{2}-\mu^{2}\right)}} d \mu=0 \text { for } n \geq 0 \text { and } m, m^{\prime}=1,2, \ldots, 2 n+1 \text { for } m \neq m^{\prime} .
$$

The integral (46) extends over the whole two-hyperboloid, i.e., $\mu$ first runs from the point $h_{3}$ to $h_{2}$ along the positive branch of $\sqrt{h_{2}^{2}-\mu^{2}}$ and then back from $h_{2}$ to $h_{3}$ along the negative branch of the same square root. Thus, we have gathered all knowledge relevant to the orthogonality properties on the surface of any constant two-hyperboloid for $v= \pm v_{s}$.

In summary, the presented methodology covers applications in different physical areas of science and modern technology, where ellipsoids, one-hyperboloids, or two-hyperboloids are involved, along with their asymptotic shapes. Some of these applications are rigorously demonstrated in Sec. IV.

\section{APPLICATION TO BOUNDARY VALUE PROBLEMS IN ELECTROSTATICS}

In order to demonstrate the applicability of our technique, we readily use the previous analysis for each coordinate surface that fits our stated problem. In fact, we will analyze two basic problems 
in the main field of electrostatics, where a two-hyperboloid of elliptic cross section and its asymptote double cone can be utilized to model an electromagnetic boundary value problem.

\section{A. A charged two-hyperboloid of non-circular cross section}

One of the basic boundary value problems for hyperboloidal harmonics involves a twohyperboloid $v= \pm v_{0}$ of elliptic cross section and perfectly conducting boundaries with the assigned potentials of $u_{2 h}=V$ on $v=v_{0}$ and $u_{2 h}=-V$ on $v=-v_{0}$, also comprising the constant potentials of the metallic area $|v|>v_{0}$. Let it be pointed out that, for symmetry, this is equivalent to a twohyperboloid of potential $V$ in front of a plane surface of potential zero. Hence, the actual domain of field calculation is $|v|<v_{0}$, wherein the case of a rotationally symmetric hyperboloid has been considered earlier in, e.g., Refs. 4 and 8 . In this particular case, every confocal two-hyperboloid $\pm v$ forms an equipotential surface. The $x_{1}=0$ plane coincides with the surface $v=0$, on which $u_{2} h$ $=0$, thus representing a symmetry plane of zero potential, whereas the field distribution varies with respect to $\rho$ and $\mu$. This symmetry requirement forces us to impose the convenient value of $x_{c}=0$ into definition (23), an ansatz to be confirmed later from the final solution.

Since the potential $u_{2 h}$ is a two-hyperboloidal harmonic function, which generates a divergencefree electric field, it can be expanded via (41), using notation (39), (40), and (42) as

$$
u_{2 h}(\rho, \mu, v)=\sum_{n=0}^{\infty} \sum_{m=1}^{2 n+1}\left[A_{n, 2 h}^{m} E_{n}^{m}(v)+B_{n, 2 h}^{m} F_{n}^{m}(v)\right] S_{n, 2 h}^{m}(\rho, \mu),
$$

for every $\rho \in\left[h_{2},+\infty\right), \mu \in\left[h_{3}, h_{2}\right]$ and, for the interval of interest, $v \in\left[-v_{0}, v_{0}\right]$. Imposing the two symmetric conditions, i.e., $u_{2 h}= \pm V$ on $v= \pm v_{0}$, relationship (47) gives two separate conditions

$$
\pm V=\sum_{n=0}^{\infty} \sum_{m=1}^{2 n+1}\left[A_{n, 2 h}^{m} E_{n}^{m}\left( \pm v_{0}\right)+B_{n, 2 h}^{m} F_{n}^{m}\left( \pm v_{0}\right)\right] S_{n, 2 h}^{m}(\rho, \mu) \text { for } \rho \in\left[h_{2},+\infty\right) \text { and } \mu \in\left[h_{3}, h_{2}\right],
$$

which, by virtue of the orthogonality relation (44), imply

$$
A_{n, 2 h}^{m}=B_{n, 2 h}^{m}=0 \text { for every } n \geq 1 \text { and } m=1,2, \ldots, 2 n+1,
$$

while the remaining constant coefficient for $n=0$ and $m=1$ secures the equalities

$$
A_{0,2 h}^{1}+B_{0,2 h}^{1} F_{0}^{1}\left(v_{0}\right)=V \text { and } A_{0,2 h}^{1}+B_{0,2 h}^{1} F_{0}^{1}\left(-v_{0}\right)=-V \text {, since } E_{0}^{1}\left( \pm v_{0}\right)=1,
$$

for $v=v_{0}$ and $v=-v_{0}$, respectively. Therein, bearing in mind that $F_{0}^{1}\left(-v_{0}\right)=-F_{0}^{1}\left(v_{0}\right)$ (see definition (23) by a simple change of variables in the relevant integral), relations (52) are manipulated to offer

$$
A_{0,2 h}^{1}=0 \text { and } B_{0,2 h}^{1}=\frac{V}{F_{0}^{1}\left(v_{0}\right)}=\frac{-V}{F_{0}^{1}\left(-v_{0}\right)} .
$$

Thus, inserting (49) and (51) into expression (47) and taking into account (42), we arrive at

$$
u_{2 h}(v)=\frac{F_{0}^{1}(v)}{F_{0}^{1}\left(v_{0}\right)} V \text { with } F_{0}^{1}(v)=\int_{0}^{v} \frac{d t}{\sqrt{h_{2}^{2}-t^{2}} \sqrt{h_{3}^{2}-t^{2}}}=-F_{0}^{1}(-v) \text { for } v \in\left[-v_{0}, v_{0}\right],
$$

verifying that $u_{2 h}$, depicted in Figure 2, varies with respect to $v$ and satisfies the initial boundary value problem. Here, we justify our symmetry requirement to set conveniently $x_{c}=0$, since $u_{2 h}(0)=0$.

The electric field $\mathbf{E}_{2 h}$, which is extended between the two sheets of the two-hyperboloid of non-circular cross section is given via the potential (52) by application of the gradient operator (13), i.e.,

$$
\mathbf{E}_{2 h}(\rho, \mu, v)=-\nabla u_{2 h}(v)=-\frac{\hat{\boldsymbol{v}}}{h_{v}} \frac{V}{F_{0}^{1}\left(v_{0}\right)} \frac{d F_{0}^{1}(v)}{d v}=-\frac{V}{h_{v} F_{0}^{1}\left(v_{0}\right) \sqrt{\left(h_{2}^{2}-v^{2}\right)\left(h_{3}^{2}-v^{2}\right)}} \hat{\boldsymbol{v}},
$$

at $\rho \in\left[h_{2},+\infty\right), \mu \in\left[h_{3}, h_{2}\right]$, and $-v_{0} \leq v \leq v_{0}$, the last equality being an immediate result of (23). 


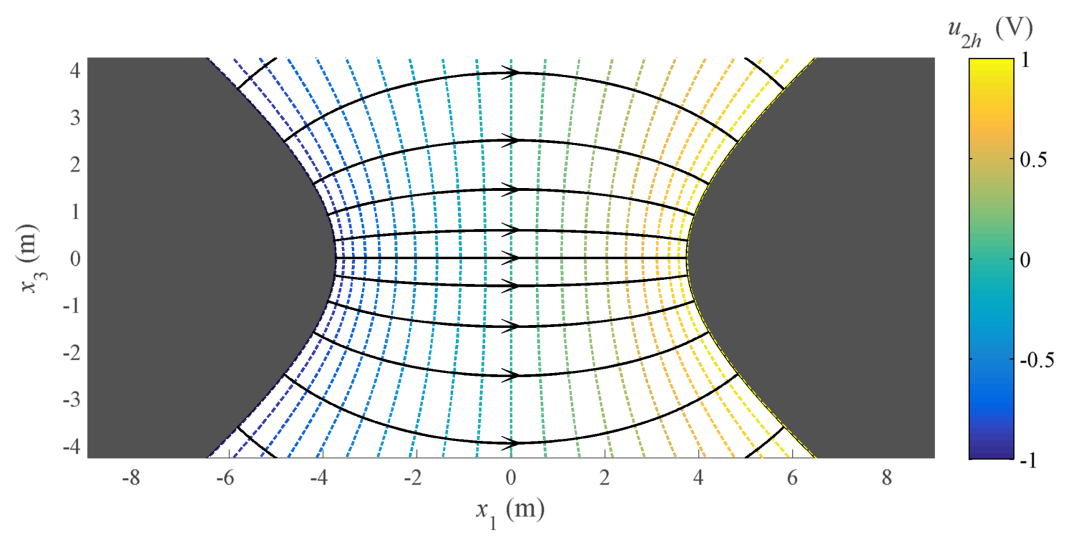

FIG. 2. Contour lines of the electric potential $u_{2} h$, vertical to the electric field lines, on $x_{1} x_{3}$-plane for $v_{0}=0.9 h_{3}$ and $V=1 \mathrm{~V}$ of elliptic two-hyperboloid for $\left(\alpha_{1}, \alpha_{2}, \alpha_{3}\right)=(5.76,4,3.2)[=] \mathrm{m}$.

The charge carried by a confocal two-hyperboloid is given by a surface integral of the charge density $\sigma_{2 h}=\varepsilon_{0} \hat{\boldsymbol{v}} \cdot \mathbf{E}_{2 h}\left(\varepsilon_{0}\right.$ being the permittivity of the vacuum) on either sheet of the hyperboloid, that is,

$$
\begin{aligned}
Q_{2 h} & =\int_{h_{3}}^{h_{2}} \int_{h_{2}}^{P} \sigma_{2 h}\left(\rho, \mu, v_{0}\right) d s_{2 h}(\rho, \mu) \\
& =\varepsilon_{0} \int_{h_{3}}^{h_{2}} \int_{h_{2}}^{P} \hat{v} \cdot \mathbf{E}_{2 h}\left(\rho, \mu, v_{0}\right) h_{\rho}\left(\rho, \mu, v_{0}\right) h_{\mu}\left(\rho, \mu, v_{0}\right) d \rho d \mu \\
& =-\frac{\varepsilon_{0} V}{F_{0}^{1}\left(v_{0}\right) \sqrt{\left(h_{2}^{2}-v_{0}^{2}\right)\left(h_{3}^{2}-v_{0}^{2}\right)}} \int_{h_{3}}^{h_{2}} \int_{h_{2}}^{P} \frac{h_{\rho}\left(\rho, \mu, v_{0}\right) h_{\mu}\left(\rho, \mu, v_{0}\right)}{h_{v}\left(\rho, \mu, v_{0}\right)} d \rho d \mu \\
& =-\frac{\varepsilon_{0} V}{F_{0}^{1}\left(v_{0}\right) \sqrt{\left(h_{2}^{2}-v_{0}^{2}\right)\left(h_{3}^{2}-v_{0}^{2}\right)}} \int_{h_{3}}^{P} \frac{h_{\rho}\left(\rho, \mu, v_{0}\right) h_{\mu}\left(\rho, \mu, v_{0}\right)}{h_{\nu}\left(\rho, \mu, v_{0}\right)} d \rho d \mu \\
& =-\frac{\varepsilon_{0} V}{F_{0}^{1}\left(v_{0}\right)} \int_{h_{3}}^{h_{2}} \int_{h_{2}}^{P} \frac{\sqrt{\rho^{2}-h_{3}^{2}} \sqrt{\rho^{2}-h_{2}^{2}} \sqrt{\mu^{2}-h_{3}^{2}} \sqrt{h_{2}^{2}-\mu^{2}}}{\sqrt{2}} d \rho d \mu,
\end{aligned}
$$

where the metric coefficients (12) have been extensively used for $v=v_{0}$ or $v=-v_{0}$. The capacitance of the two-hyperboloid is finally given by the quantity $Q_{2 h} / 2 V_{2 h}$, where $V_{2 h}$ being the volume of the two-hyperboloid, cut by any confocal ellipsoid $\rho=$ constant. Next, we observe that the total charge remains finite provided that the two sheets of the hyperboloid are cut by the ellipsoid at a finite distance $P$. At the edge of the hyperboloid, the fringing fields occurring along the edge are ignored. Thus, the obtained expression for the capacitance is an approximation, which is improving as the hyperboloid becomes larger. Finally, we mention that the normalized surface charge density on a two-hyperboloid of prescribed dimensions is the largest at the apex and along the upper and lower rim of the hyperboloid, where the radius of the curvature is the smallest. The behavior of the magnitude of the electric field $\left|\mathbf{E}_{2 h}\right|$ from (53) at the cross section $x_{2}=0$ and of the absolute value of the charge density $\left|\sigma_{2 h}\right|$ in three dimensions is sketched within Figures 3 and 4, respectively, being demonstrated for the cases of both a two-hyperboloid (see Figures 3(a) and 4(a)) and an approximated double-cone (see Figures 3(b) and 4(b)) of elliptic cross section.

The degenerate case of a two-hyperboloid of circular cross section is obtained at the special case when $h_{2}=h_{3} \equiv h$, where the corresponding results are easily derived from (52)-(54) and by virtue 
(a)

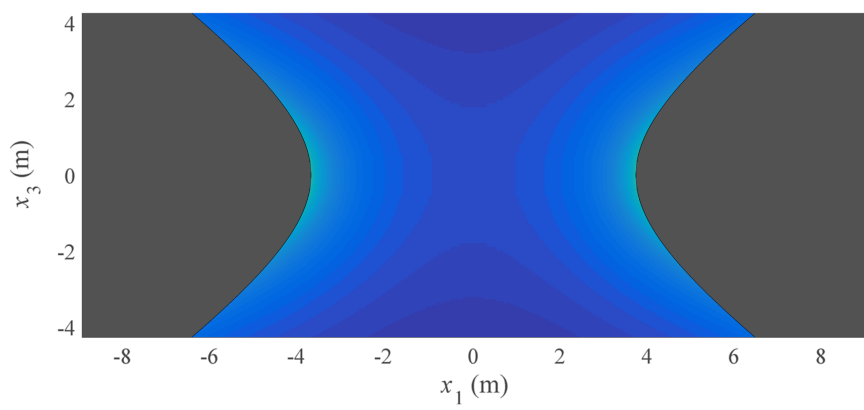

(b)

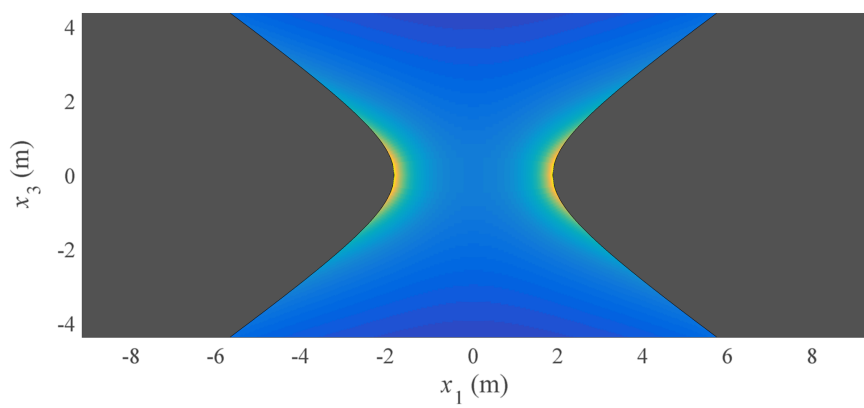

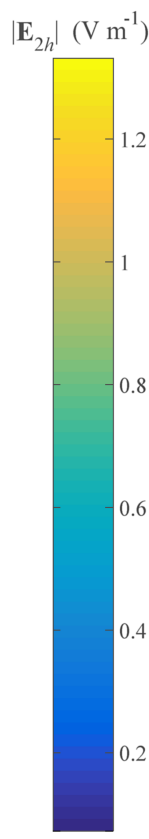

FIG. 3. Electric field strength $\left|\mathbf{E}_{2 h}\right|$ on $x_{1} x_{3}$-plane for $v_{0}=0.9 h_{3}$ and $V=1 \mathrm{~V}$ of elliptic (a) two-hyperboloid for $\left(\alpha_{1}, \alpha_{2}, \alpha_{3}\right)$ $=(5.76,4,3.2)[=] \mathrm{m}$ and (b) approximated double-cone for a smaller reference ellipsoid with semi-axes $\left(\alpha_{1}, \alpha_{2}, \alpha_{3}\right)=(2.88$, $2,1.6)[=] \mathrm{m}$.

of the Lamé solutions (23). Indeed, in this case

$$
\lim _{h_{2}, h_{3} \rightarrow h} F_{0}^{1}(v)=\lim _{h_{2}, h_{3} \rightarrow h} \int_{0}^{v} \frac{d t}{\sqrt{h_{2}^{2}-t^{2}} \sqrt{h_{3}^{2}-t^{2}}}=\int_{0}^{v} \frac{d t}{h^{2}-t^{2}}=\frac{1}{h} \tanh ^{-1}\left(\frac{v}{h}\right) \text { for } v \in\left[-v_{0}, v_{0}\right],
$$

yielding to a potential, which results from (52), i.e.,

$$
\lim _{h_{2}, h_{3} \rightarrow h} u_{2 h}(v)=\frac{\tanh ^{-1}(v / h)}{\tanh ^{-1}\left(v_{0} / h\right)} V, \text { where } \tanh ^{-1}\left(\frac{v}{h}\right)=-\tanh ^{-1}\left(-\frac{v}{h}\right) \text { for every }-v_{0} \leq v \leq v_{0} .
$$

The electric field $\mathbf{E}_{2 h}$, the charge $Q_{2 h}$, and the capacitance in this limiting case are straightforwardly calculated once (56) is implied. Moreover, as the main reference ellipsoid becomes smaller, the two-hyperboloid is approached by the corresponding asymptotic elliptical or circular double-cone.
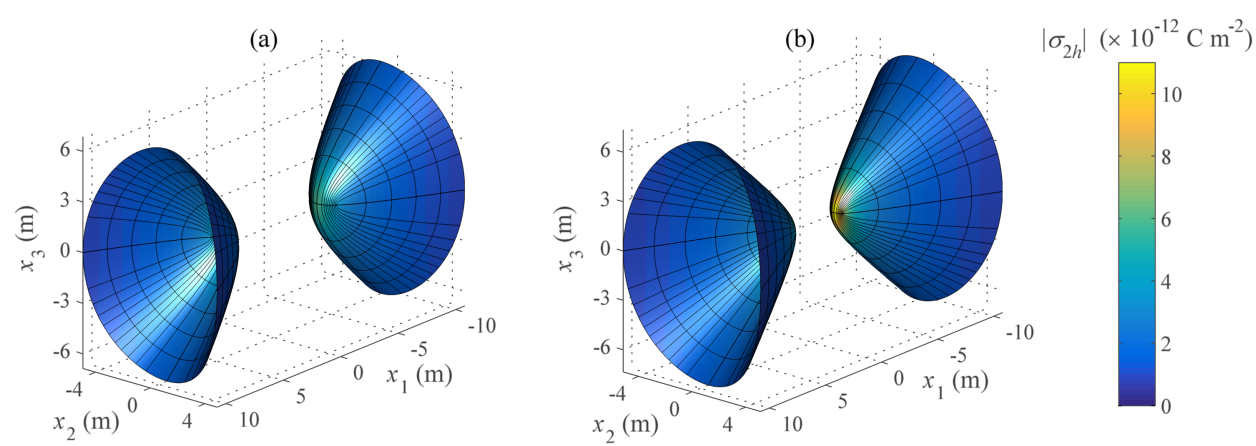

FIG. 4. Charge density $\left|\sigma_{2 h}\right|$ for $v_{0}=0.9 h_{3}, \varepsilon_{0}=8.854 \times 10^{-12} \mathrm{~F} / \mathrm{m}$, and $V=1 \mathrm{~V}$ of elliptic (a) two-hyperboloid for $\left(\alpha_{1}\right.$, $\left.\alpha_{2}, \alpha_{3}\right)=(5.76,4,3.2)$ [=]m and (b) approximated double-cone for a smaller reference ellipsoid with semi-axes $\left(\alpha_{1}, \alpha_{2}, \alpha_{3}\right)$ $=(2.88,2,1.6)[=] \mathrm{m}$. 
Therefore, the goal of evaluating the electric field near a corner or edge has been achieved, where obviously, for the case of a double-cone, the electric field strength and the charge density are increased at a sharper edge. Besides, it is well known ${ }^{1}$ that the density of the charge is an increasing function of the local curvature, concentrating almost entirely on the tips.

\section{B. A non-circular metal two-hyperboloid subject to an electric and magnetic plane wave}

We continue with a slightly more complicated application and we turn to the case of a twohyperboloid of elliptic cross section, where the two sheets that are set at $v=v_{0}>0$ and $v=-v_{0}$ behave as perfect conductors. The system is subject to constant electric $\mathbf{E}_{2 h}^{i}$ and magnetic $\mathbf{H}_{2 h}^{i}$ incident fields, which are mutually perpendicular, and also perpendicular to the direction of propagation, being the approximation of a slowly varying wave (of long wavelength compared to the dimensions of the gap of the cone) and applied transversely to the axis of the two-sided hyperboloid, that is,

$$
\mathbf{E}_{2 h}^{i}=-\nabla u_{2 h}^{i}(\rho, \mu, v)=E_{2 h}^{i, \pm} \hat{\boldsymbol{x}}_{\mathbf{3}}=E_{2 h}^{i, \pm}\left[\left(\hat{\boldsymbol{x}}_{3} \cdot \hat{\boldsymbol{\rho}}\right) \hat{\boldsymbol{\rho}}+\left(\hat{\boldsymbol{x}}_{3} \cdot \hat{\boldsymbol{\mu}}\right) \hat{\boldsymbol{\mu}}+\left(\hat{\boldsymbol{x}}_{3} \cdot \hat{\boldsymbol{v}}\right) \hat{\boldsymbol{v}}\right]
$$

and

$$
\mathbf{H}_{2 h}^{i}=-\nabla w_{2 h}^{i}(\rho, \mu, v)=H_{2 h}^{i, \pm} \hat{\boldsymbol{x}}_{\mathbf{3}}=H_{2 h}^{i, \pm}\left[\left(\hat{\boldsymbol{x}}_{3} \cdot \hat{\boldsymbol{\rho}}\right) \hat{\boldsymbol{\rho}}+\left(\hat{\boldsymbol{x}}_{3} \cdot \hat{\boldsymbol{\mu}}\right) \hat{\boldsymbol{\mu}}+\left(\hat{\boldsymbol{x}}_{3} \cdot \hat{\boldsymbol{v}}\right) \hat{\boldsymbol{v}}\right],
$$

which are designated through the primary electric potential function

$$
u_{2 h}^{i}(\rho, \mu, v)=-E_{2 h}^{i, \pm} x_{3}=-E_{2 h}^{i, \pm} \frac{\sqrt{\rho^{2}-h_{2}^{2}} \sqrt{h_{2}^{2}-\mu^{2}} \sqrt{h_{2}^{2}-v^{2}}}{h_{1} h_{2}}=-E_{2 h}^{i, \pm} \frac{\mathrm{E}_{1,2 h}^{3}(\rho, \mu, v)}{h_{1} h_{2}}
$$

and the primary magnetic potential function

$$
w_{2 h}^{i}(\rho, \mu, v)=-H_{2 h}^{i, \pm} x_{3}=-H_{2 h}^{i, \pm} \frac{\sqrt{\rho^{2}-h_{2}^{2}} \sqrt{h_{2}^{2}-\mu^{2}} \sqrt{h_{2}^{2}-v^{2}}}{h_{1} h_{2}}=-H_{2 h}^{i, \pm} \frac{\mathrm{E}_{1,2 h}^{3}(\rho, \mu, v)}{h_{1} h_{2}},
$$

respectively, written for $\mathrm{E}_{1,2 h}^{3}(\rho, \mu, v)=E_{1}^{3}(v) S_{1,2 h}^{3}(\rho, \mu)$, having used coordinate variable (4), Lamé functions (18)-(21) with (22), as well as (39) with (42), where

$$
E_{2 h}^{i, \pm}=\left\{\begin{array}{l}
-E_{2 h}^{i}, v \in\left[-v_{0}, 0\right) \\
E_{2 h}^{i}, v \in\left[0, v_{0}\right]
\end{array} \text { and } H_{2 h}^{i, \pm}=\left\{\begin{array}{l}
-H_{2 h}^{i}, v \in\left[-v_{0}, 0\right) \\
H_{2 h}^{i}, v \in\left[0, v_{0}\right]
\end{array}\right.\right.
$$

represent the kind of incidence we choose for reasons of symmetry and in order to be compatible with the interval of variation of variable $v$ within (59) and (60), while for $v=0$, the primary fields are assumed to attain the positive direction. The scattering region is confined by $\rho \in\left[h_{2},+\infty\right), \mu \in\left[h_{3}\right.$, $\left.h_{2}\right]$ and limited to the interval $-v_{0} \leq v \leq v_{0}$, whereas this domain of scattering action is being defined as $\Omega$. The incident fields are perturbed by the solid targets for $v= \pm v_{0}$, producing the corresponding electromagnetic scattered fields $\mathbf{E}_{2 h}^{s}$ and $\mathbf{H}_{2 h}^{s}$. Those are offered in terms of the scattered electric potential $u_{2 h}^{s}$ and magnetic potential $w_{2 h}^{s}$, which are assumed to have identical expansions with that in (41). Consequently, for every $(\rho, \mu, v) \in \Omega$, we obtain

$$
\mathbf{E}_{2 h}^{s}(\rho, \mu, v)=-\nabla u_{2 h}^{s}(\rho, \mu, v)=-\nabla\left\{\sum_{n=0}^{\infty} \sum_{m=1}^{2 n+1}\left[A_{n, 2 h}^{m, u} \mathrm{E}_{n, 2 h}^{m}(\rho, \mu, v)+B_{n, 2 h}^{m, u} \mathrm{~F}_{n, 2 h}^{m}(\rho, \mu, v)\right]\right\}
$$

and

$$
\mathbf{H}_{2 h}^{s}(\rho, \mu, v)=-\nabla w_{2 h}^{s}(\rho, \mu, v)=-\nabla\left\{\sum_{n=0}^{\infty} \sum_{m=1}^{2 n+1}\left[A_{n, 2 h}^{m, w} \mathrm{E}_{n, 2 h}^{m}(\rho, \mu, v)+B_{n, 2 h}^{m, w} \mathrm{~F}_{n, 2 h}^{m}(\rho, \mu, v)\right]\right\},
$$

where the harmonic eigenfunctions $\mathrm{E}_{n, 2 h}^{m}$ and $\mathrm{F}_{n, 2 h}^{m}$ are provided in (39) and (40), respectively, with respect to (42). The total fields,

$$
\mathbf{E}_{2 h}^{t}(\rho, \mu, v)=\mathbf{E}_{2 h}^{i}+\mathbf{E}_{2 h}^{s}(\rho, \mu, v) \text { and } \mathbf{H}_{2 h}^{t}(\rho, \mu, v)=\mathbf{H}_{2 h}^{i}+\mathbf{H}_{2 h}^{s}(\rho, \mu, v) \text { for }(\rho, \mu, v) \in \Omega,
$$

are given as the sum of the incident and the scattered fields, all being divergence-free. 
The constant coefficients $A_{n, 2 h}^{m, u}, B_{n, 2 h}^{m, u}$ and $A_{n, 2 h}^{m, w}, B_{n, 2 h}^{m, w}$ must be determined from the proper perfectly conducting boundary conditions on the surface of the metallic hyperboloid of two sheets at $v= \pm v_{0}$, i.e.,

$$
\hat{\boldsymbol{v}} \times \mathbf{E}_{2 h}^{t}\left(\rho, \mu, \pm v_{0}\right)=\mathbf{0} \text { and } \hat{\boldsymbol{v}} \cdot \mathbf{H}_{2 h}^{t}\left(\rho, \mu, \pm v_{0}\right)=0 \text { for every } \rho \in\left[h_{2},+\infty\right), \mu \in\left[h_{3}, h_{2}\right],
$$

in terms of the outward unit normal vector $\hat{v}$, whereas the tangential components of the total electric field and the normal component of the total magnetic field cancel on $v= \pm v_{0}$. The nature of the incident potentials (59) and (60) is inherited by the scattered ones, that is, due to orthogonality (44) and in terms of (65), the only surviving eigenfunctions are for $n=1$ and $m=3$, bearing in mind that coefficients $A_{0,2 h}^{1, u}$ and $A_{0,2 h}^{1, w}$ that do not appear in (62) and (63), refer to the constant counterparts of the harmonic potentials that generate the scattered electromagnetic fields and have been arbitrarily set to zero, without losing uniqueness. Indeed, since the aforementioned fields appear under the gradient operator within (62) and (63), the relevant eigenfunctions $\mathrm{E}_{0,2 h}^{1}$ do not contribute to the solution, hence we chose to eliminate this part without loss of generality. Therefore, we readily conclude to

$$
A_{n, 2 h}^{m, u}=A_{n, 2 h}^{m, w}=B_{n, 2 h}^{m, u}=B_{n, 2 h}^{m, w}=0 \text { for every } n \geq 0 \text { and } m=1,2, \ldots, 2 n+1 \text { with } n \neq 1 \text { and } m \neq 3,
$$

while the rest of the constants are evaluated as it is presented in the sequel. First, substituting (66) into (62) and (63), the potentials

$u_{2 h}^{s}(\rho, \mu, v)=A_{1,2 h}^{3, u} \mathrm{E}_{1,2 h}^{3}(\rho, \mu, v)+B_{1,2 h}^{3, u} \mathrm{~F}_{1,2 h}^{3}(\rho, \mu, v)=\left[A_{1,2 h}^{3, u} E_{1}^{3}(v)+B_{1,2 h}^{3, u} F_{1}^{3}(v)\right] S_{1,2 h}^{3}(\rho, \mu)$

and

$$
w_{2 h}^{s}(\rho, \mu, v)=A_{1,2 h}^{3, w} \mathrm{E}_{1,2 h}^{3}(\rho, \mu, v)+B_{1,2 h}^{3, w} \mathrm{~F}_{1,2 h}^{3}(\rho, \mu, v)=\left[A_{1,2 h}^{3, w} E_{1}^{3}(v)+B_{1,2 h}^{3, w} F_{1}^{3}(v)\right] S_{1,2 h}^{3}(\rho, \mu),
$$

for every $(\rho, \mu, v) \in \Omega$ and in addition to (13), produce the electric and the magnetic fields

$$
\begin{aligned}
\mathbf{E}_{2 h}^{s}(\rho, \mu, v)= & -\nabla\left[A_{1,2 h}^{3, u} \mathrm{E}_{1,2 h}^{3}(\rho, \mu, v)+B_{1,2 h}^{3, u} \mathrm{~F}_{1,2 h}^{3}(\rho, \mu, v)\right] \\
= & -\nabla\left\{\left[A_{1,2 h}^{3, u} E_{1}^{3}(v)+B_{1,2 h}^{3, u} F_{1}^{3}(v)\right] S_{1,2 h}^{3}(\rho, \mu)\right\} \\
= & -\nabla\left\{\left[A_{1,2 h}^{3, u} E_{1}^{3}(v)+B_{1,2 h}^{3, u} F_{1}^{3}(v)\right] E_{1}^{3}(\rho) E_{1}^{3}(\mu)\right\} \\
= & -\left[\left(\frac{\hat{\boldsymbol{\rho}}}{h_{\rho}} E_{1}^{3 \prime}(\rho) E_{1}^{3}(\mu)+\frac{\hat{\boldsymbol{\mu}}}{h_{\mu}} E_{1}^{3}(\rho) E_{1}^{3{ }^{\prime}}(\mu)\right)\left(A_{1,2 h}^{3, u} E_{1}^{3}(v)+B_{1,2 h}^{3, u} F_{1}^{3}(v)\right)\right. \\
& \left.+\frac{\hat{v}}{h_{v}} E_{1}^{3}(\rho) E_{1}^{3}(\mu)\left(A_{1,2 h}^{3, u} E_{1}^{3 \prime}(v)+B_{1,2 h}^{3, u} F_{1}^{3^{\prime}}(v)\right)\right] \text { for every }(\rho, \mu, v) \in \Omega
\end{aligned}
$$

and

$$
\begin{aligned}
\mathbf{H}_{2 h}^{s}(\rho, \mu, v)= & -\nabla\left[A_{1,2 h}^{3, w} \mathrm{E}_{1,2 h}^{3}(\rho, \mu, v)+B_{1,2 h}^{3, w} \mathrm{~F}_{1,2 h}^{3}(\rho, \mu, v)\right] \\
= & -\nabla\left\{\left[A_{1,2 h}^{3, w} E_{1}^{3}(v)+B_{1,2 h}^{3, w} F_{1}^{3}(v)\right] S_{1,2 h}^{3}(\rho, \mu)\right\} \\
= & -\nabla\left\{\left[A_{1,2 h}^{3, w} E_{1}^{3}(v)+B_{1,2 h}^{3, w} F_{1}^{3}(v)\right] E_{1}^{3}(\rho) E_{1}^{3}(\mu)\right\} \\
= & -\left[\left(\frac{\hat{\boldsymbol{\rho}}}{h_{\rho}} E_{1}^{3^{\prime}}(\rho) E_{1}^{3}(\mu)+\frac{\hat{\boldsymbol{\mu}}}{h_{\mu}} E_{1}^{3}(\rho) E_{1}^{3 \prime}(\mu)\right)\left(A_{1,2 h}^{3, w} E_{1}^{3}(v)+B_{1,2 h}^{3, w} F_{1}^{3}(v)\right)\right. \\
& \left.+\frac{\hat{\boldsymbol{v}}}{h_{v}} E_{1}^{3}(\rho) E_{1}^{3}(\mu)\left(A_{1,2 h}^{3, w} E_{1}^{3{ }^{\prime}}(v)+B_{1,2 h}^{3, w} F_{1}^{3^{\prime}}(v)\right)\right] \text { for every }(\rho, \mu, v) \in \Omega,
\end{aligned}
$$

correspondingly, where the prime signifies the derivative with respect to the argument, while definitions (39), (40), and (42) have been implied. We, now, reinforce the condition for the total electric field on both the surfaces of the two-hyperboloid at $v= \pm v_{0}$, which by virtue of (64), gives

$$
\hat{\boldsymbol{v}} \times\left[\mathbf{E}_{2 h}^{i}+\mathbf{E}_{2 h}^{s}\left(\rho, \mu, \pm \nu_{0}\right)\right]=\mathbf{0} \text { for every } \rho \in\left[h_{2},+\infty\right) \text { and } \mu \in\left[h_{3}, h_{2}\right] \text {. }
$$


Combining (71) with (57) and (69), as well as the fact that the ellipsoidal system $\hat{\rho}, \hat{\boldsymbol{v}}, \hat{\boldsymbol{\mu}}$ is dextral, we obtain after some straightforward calculations, based to (4) and (15),

$\left[\hat{\boldsymbol{\rho}} \frac{E_{1}^{3}(\rho) E_{1}^{3^{\prime}}(\mu)}{h_{\mu}}-\hat{\boldsymbol{\mu}} \frac{E_{1}^{3^{\prime}}(\rho) E_{1}^{3}(\mu)}{h_{\rho}}\right]\left\{E_{2 h}^{i, \pm} \frac{E_{1}^{3}\left( \pm v_{0}\right)}{h_{1} h_{2}}-\left[A_{1,2 h}^{3, u} E_{1}^{3}\left( \pm v_{0}\right)+B_{1,2 h}^{3, u} F_{1}^{3}\left( \pm v_{0}\right)\right]\right\}=\mathbf{0}$,

for every $\rho \in\left[h_{2},+\infty\right)$ and $\mu \in\left[h_{3}, h_{2}\right]$. The latter one is satisfied only when the constant quantity among the braces is zero, providing us with the relation

$$
A_{1,2 h}^{3, u} E_{1}^{3}\left( \pm v_{0}\right)+B_{1,2 h}^{3, u} F_{1}^{3}\left( \pm v_{0}\right)=E_{2 h}^{i, \pm} \frac{E_{1}^{3}\left( \pm v_{0}\right)}{h_{1} h_{2}},
$$

which, by virtue of (61), offers the following two independent relations for $v=v_{0}$ and $v=-v_{0}$ :

$A_{1,2 h}^{3, u} E_{1}^{3}\left(v_{0}\right)+B_{1,2 h}^{3, u} F_{1}^{3}\left(v_{0}\right)=E_{2 h}^{i} \frac{E_{1}^{3}\left(v_{0}\right)}{h_{1} h_{2}}$ and $A_{1,2 h}^{3, u} E_{1}^{3}\left(-v_{0}\right)+B_{1,2 h}^{3, u} F_{1}^{3}\left(-v_{0}\right)=-E_{2 h}^{i} \frac{E_{1}^{3}\left(-v_{0}\right)}{h_{1} h_{2}}$.

To solve system (74) and obtain the solution, we invoke the ellipsoidal functions from the class $\mathrm{M}$ of definitions (20) and (23), admitting

$E_{1}^{3}(v)=\sqrt{h_{2}^{2}-v^{2}}=E_{1}^{3}(-v)$ and $F_{1}^{3}(v)=E_{1}^{3}(v) \int_{0}^{v} \frac{d t}{\left[E_{1}^{3}(t)\right]^{2} \sqrt{h_{2}^{2}-t^{2}} \sqrt{h_{3}^{2}-t^{2}}}=-F_{1}^{3}(-v)$,

for every value of $-v_{0} \leq v \leq v_{0}$, setting $x_{c}=0$ without loss of generality, in order for relationship (74) to be easily solvable. Hence, in view of (75), conditions (74) reveal that

$$
A_{1,2 h}^{3, u}=0 \text { and } B_{1,2 h}^{3, u}=E_{2 h}^{i} \frac{1}{h_{1} h_{2}} \frac{E_{1}^{3}\left(v_{0}\right)}{F_{1}^{3}\left(v_{0}\right)},
$$

via the magnitude of the primary electric field $E_{2 h}^{i}$. Imposing (76) into the electric potential function $u_{2 h}^{s}$ of the electric field (67), we readily arrive at

$$
u_{2 h}^{s}(\rho, \mu, v)=E_{2 h}^{i} \frac{E_{1}^{3}\left(v_{0}\right)}{h_{1} h_{2}}\left(\frac{F_{1}^{3}(v)}{F_{1}^{3}\left(v_{0}\right)}\right) E_{1}^{3}(\rho) E_{1}^{3}(\mu) \text { for every }(\rho, \mu, v) \in \Omega,
$$

from which the scattered electric field $\mathbf{E}_{2 h}^{s}$ is evaluated directly via (69), whose measure is being demonstrated in Figure 5, while the corresponding strength of the total electric field, i.e., $\left|\mathbf{E}_{2 h}^{t}\right|$ is depicted within Figure 6. In the sequel, we apply the magnetic boundary condition (65), which reads

$$
\hat{\boldsymbol{v}} \cdot\left[\mathbf{H}_{2 h}^{i}+\mathbf{H}_{2 h}^{s}\left(\rho, \mu, \pm v_{0}\right)\right]=0 \text { for every } \rho \in\left[h_{2},+\infty\right) \text { and } \mu \in\left[h_{3}, h_{2}\right],
$$

where together with (15), (58), and (70), is transformed to

$$
\frac{E_{1}^{3}(\rho) E_{1}^{3}(\mu)}{h_{\nu}}\left[H_{2 h}^{i, \pm} \frac{E_{1}^{3^{\prime}}\left( \pm v_{0}\right)}{h_{1} h_{2}}-\left(A_{1,2 h}^{3, w} E_{1}^{3^{\prime}}\left( \pm v_{0}\right)+B_{1,2 h}^{3, w} F_{1}^{3^{\prime}}\left( \pm v_{0}\right)\right)\right]=0,
$$

for every $\rho \in\left[h_{2},+\infty\right)$ and $\mu \in\left[h_{3}, h_{2}\right]$. Solving (79), we conclude that

$$
A_{1,2 h}^{3, w} E_{1}^{3^{\prime}}\left( \pm v_{0}\right)+B_{1,2 h}^{3, w} F_{1}^{3^{\prime}}\left( \pm v_{0}\right)=H_{2 h}^{i, \pm} \frac{E_{1}^{3^{\prime}}\left( \pm v_{0}\right)}{h_{1} h_{2}}
$$

where with respect to (61) describes the two different conditions

$$
A_{1,2 h}^{3, w} E_{1}^{3^{\prime}}\left(v_{0}\right)+B_{1,2 h}^{3, w} F_{1}^{3^{\prime}}\left(v_{0}\right)=H_{2 h}^{i} \frac{E_{1}^{3^{\prime}}\left(v_{0}\right)}{h_{1} h_{2}} \text { and } A_{1,2 h}^{3, w} E_{1}^{3^{\prime}}\left(-v_{0}\right)+B_{1,2 h}^{3, w} F_{1}^{3^{\prime}}\left(-v_{0}\right)=-H_{2 h}^{i} \frac{E_{1}^{3^{\prime}}\left(-v_{0}\right)}{h_{1} h_{2}},
$$

where from notation (75), we readily arrive at

$$
E_{1}^{3^{\prime}}(v)=-\frac{v}{\sqrt{h_{2}^{2}-v^{2}}}=-E_{1}^{3^{\prime}}(-v) \text { for every }-v_{0} \leq v \leq v_{0}
$$


(a)
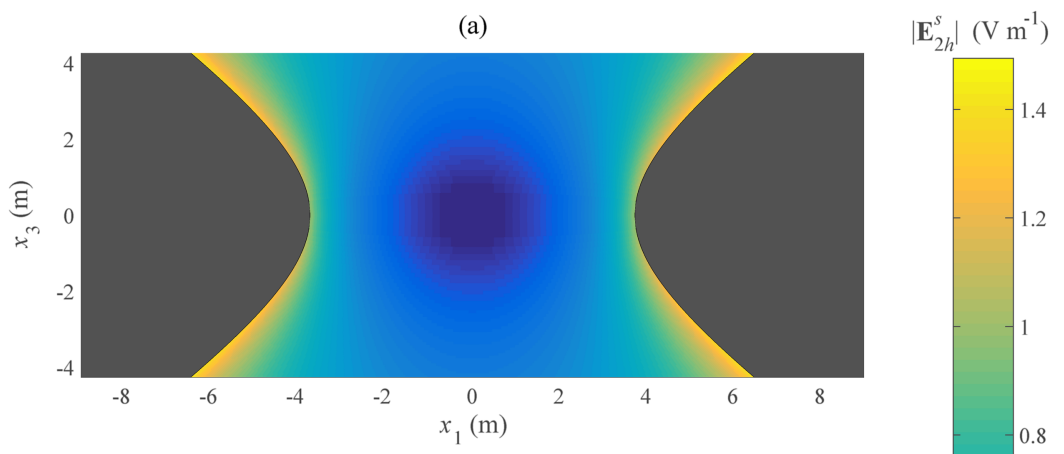

(b)

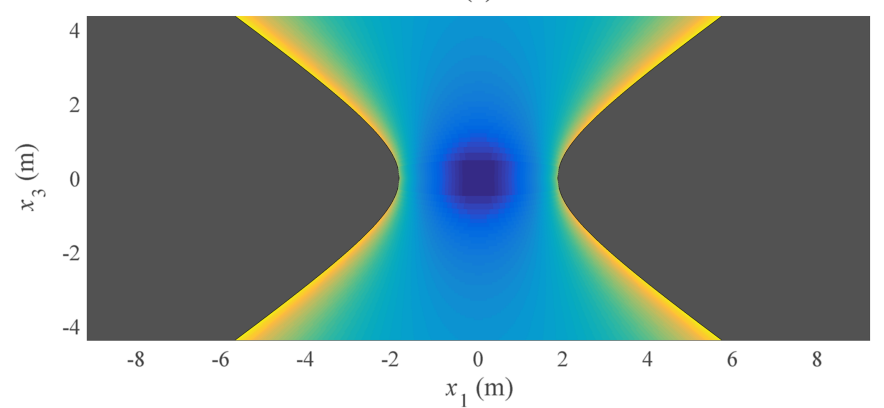

FIG. 5. Scattered electric field strength $\left|\mathbf{E}_{2 h}^{s}\right|$ on $x_{1} x_{3}$-plane for $v_{0}=0.9 h_{3}$ and $E_{2 h}^{i}=1 V$ for an elliptic cross-sectional (a) two-hyperboloid for $\left(\alpha_{1}, \alpha_{2}, \alpha_{3}\right)=(5.76,4,3.2)[=] \mathrm{m}$ and (b) approximated double-cone for a smaller reference ellipsoid with semi-axes $\left(\alpha_{1}, \alpha_{2}, \alpha_{3}\right)=(2.88,2,1.6)[=] \mathrm{m}$.

and

$$
F_{1}^{3^{\prime}}(v)=E_{1}^{3^{\prime}}(v) \int_{0}^{v} \frac{d t}{\left[E_{1}^{3}(t)\right]^{2} \sqrt{h_{2}^{2}-t^{2}} \sqrt{h_{3}^{2}-t^{2}}}+\frac{1}{\left[E_{1}^{3}(v)\right]^{2} \sqrt{h_{3}^{2}-v^{2}}}=F_{1}^{3^{\prime}}(-v) \text { for }-v_{0} \leq v \leq v_{0}
$$

Consequently, in view of (82) and (83), relations (81) comprise

$$
A_{1,2 h}^{3, w}=0 \text { and } B_{1,2 h}^{3, w}=H_{2 h}^{i} \frac{1}{h_{1} h_{2}} \frac{E_{1}^{3^{\prime}}\left(v_{0}\right)}{F_{1}^{3^{\prime}}\left(v_{0}\right)},
$$

written as a function of the magnitude of the incident magnetic field $H_{2 h}^{i}$. Entering result (84) into the magnetic potential function $w_{2 h}^{s}$ of the magnetic field (68), we are led to

$$
w_{2 h}^{s}(\rho, \mu, v)=H_{2 h}^{i} \frac{E_{1}^{3^{\prime}}\left(v_{0}\right)}{h_{1} h_{2}}\left(\frac{F_{1}^{3}(v)}{F_{1}^{3^{\prime}}\left(v_{0}\right)}\right) E_{1}^{3}(\rho) E_{1}^{3}(\mu) \text { for every }(\rho, \mu, v) \in \Omega,
$$

from which the scattered magnetic field $\mathbf{H}_{2 h}^{s}$ is immediately calculated via (70). Both the electric (77) and the magnetic (85) fields require the utilization of the relative surface two-hyperboloidal harmonic eigenfunctions,

$$
S_{1,2 h}^{3}(\rho, \mu)=E_{1}^{3}(\rho) E_{1}^{3}(\mu)=\sqrt{\rho^{2}-h_{2}^{2}} \sqrt{h_{2}^{2}-\mu^{2}} \text { for every } \rho \in\left[h_{2},+\infty\right) \text { and } \mu \in\left[h_{3}, h_{2}\right],
$$

of the first degree and of the third order.

In the special case where $h_{2}=h_{3} \equiv h$, the degenerate case of a two-hyperboloid of circular cross section is obtained, where the corresponding results of the electromagnetic potentials are obtained from (77) and (85). However, the most important reduced case is that involving a double cone of circular cross section, where in addition to the restriction mentioned above, large values of the 
(a)
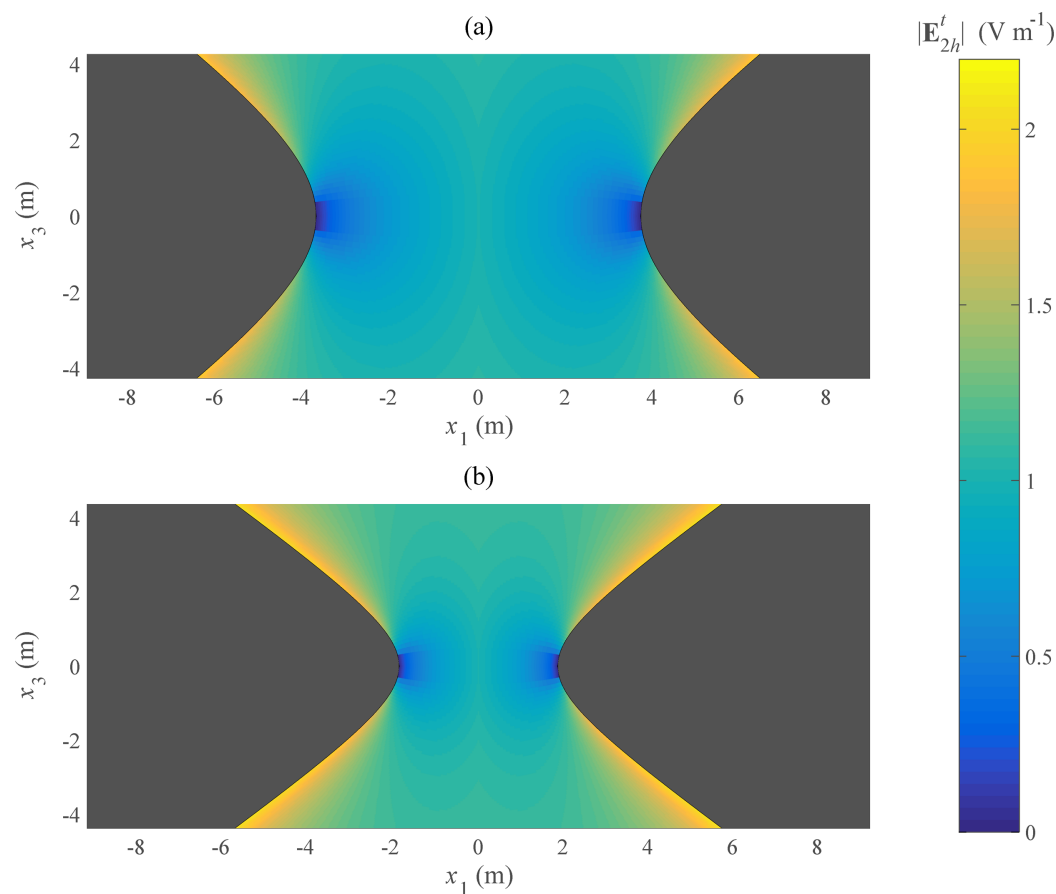

FIG. 6. Total electric field strength $\left|\mathbf{E}_{2 h}^{t}\right|$ on $x_{1} x_{3}$-plane for $v_{0}=0.9 h_{3}$ and $E_{2 h}^{i}=1 V$ for an elliptic cross-sectional (a) twohyperboloid for $\left(\alpha_{1}, \alpha_{2}, \alpha_{3}\right)=(5.76,4,3.2)[=] \mathrm{m}$ and (b) approximated double-cone for a smaller reference ellipsoid with semi-axes $\left(\alpha_{1}, \alpha_{2}, \alpha_{3}\right)=(2.88,2,1.6)[=] \mathrm{m}$.

Cartesian coordinates $x_{1}, x_{2}$, and $x_{3}$ are adequate to approximate this interesting case, which by the way represent one of the coordinate surfaces in the spherical coordinate system $(r, \theta, \varphi)$ for $r \geq 0$ and $\varphi \in[0,2 \pi)$. Furthermore, the cone is defined by the surface $\theta=\theta_{0}=$ constant in the upper half-space and by $\pi-\theta_{0}$ in the lower half-space, where in accordance to the two-hyperboloidal problem, the region of scattering is limited outside the cone, i.e., for $\theta_{0} \leq \theta \leq \pi-\theta_{0}$, defined as $W$. Our task, then, is to reduce the results (77) and (85) from the two-hyperboloidal of elliptic cross section geometry to the double-cone of circular cross section. This work requires a careful limiting process, since it is obvious that several indeterminacies appear as we analytically move from the ellipsoidal system to the spherical one, since $h_{1}=h_{2}=h_{3}=0$, meaning that all the semifocal distances of the ellipsoid recede to the origin. To this end, we use the limits ${ }^{6}$

$$
\rho\left(\equiv E_{1}^{1}(\rho)\right)=\sqrt{\rho^{2}-h_{3}^{2}}\left(\equiv E_{1}^{2}(\rho)\right)=\sqrt{\rho^{2}-h_{2}^{2}}\left(\equiv E_{1}^{3}(\rho)\right) \rightarrow r \text { for } \rho \in\left[h_{2},+\infty\right) \text { and } r \geq 0,
$$

regarding the radial component, while

$$
\begin{gathered}
\frac{\mu \nu}{h_{2} h_{3}} \equiv \frac{E_{1}^{1}(\mu) E_{1}^{1}(v)}{h_{2} h_{3}} \rightarrow \cos \theta, \\
\frac{\sqrt{\mu^{2}-h_{3}^{2}} \sqrt{h_{3}^{2}-v^{2}}}{h_{1} h_{3}} \equiv \frac{E_{1}^{2}(\mu) E_{1}^{2}(v)}{h_{1} h_{3}} \rightarrow \sin \theta \cos \varphi,
\end{gathered}
$$

and

$$
\frac{\sqrt{h_{2}^{2}-\mu^{2}} \sqrt{h_{2}^{2}-v^{2}}}{h_{1} h_{2}} \equiv \frac{E_{1}^{3}(\mu) E_{1}^{3}(v)}{h_{1} h_{2}} \rightarrow \sin \theta \sin \varphi,
$$

for every value of $\mu \in\left[h_{3}, h_{2}\right]$ and $v \in\left[-v_{0}, v_{0}\right]$, as well as $\varphi \in[0,2 \pi)$ and $\theta \in\left[\theta_{0}, \pi-\theta_{0}\right]$, concerning the angular dependence. But, also the exterior Lamé functions of the $v$-variable used in this project 
are approached by the associated Legendre functions of the second kind ${ }^{9,10}$ at the spherical limit, meaning

$$
F_{1}^{1}(v) \rightarrow Q_{1}^{-1}(\cos \theta), F_{1}^{2}(v) \rightarrow Q_{1}^{0}(\cos \theta) \text { and } F_{1}^{3}(v) \rightarrow Q_{1}^{1}(\cos \theta),
$$

for every $v \in\left[-v_{0}, v_{0}\right]$ and $\theta \in\left[\theta_{0}, \pi-\theta_{0}\right]$. Using (72)-(76), we may easily arrive at the double-cone limits

$$
\lim _{2 h \rightarrow d c} u_{2 h}^{s}(r, \theta, \varphi)=E_{2 h}^{i} \sin \theta_{0}\left(\frac{Q_{1}^{1}(\cos \theta)}{Q_{1}^{1}\left(\cos \theta_{0}\right)}\right) r \sin \varphi \text { for every }(r, \theta, \varphi) \in W,
$$

for the harmonic electric potential and

$$
\lim _{2 h \rightarrow d c} w_{2 h}^{s}(r, \theta, \varphi)=H_{2 h}^{i} \cos \theta_{0}\left(\frac{Q_{1}^{1}(\cos \theta)}{Q_{1}^{1^{\prime}}\left(\cos \theta_{0}\right)}\right) r \sin \varphi \text { for every }(r, \theta, \varphi) \in W,
$$

for the harmonic magnetic potential, respectively. These limits coincide with the analytical expressions for the problem of electromagnetic scattering by a double-cone of spherical cross section. It is clear that the electromagnetic fields for the particular situation can be easily derived via (69) and (70) via the limiting processes

$$
\lim _{2 h \rightarrow d c} \mathbf{E}_{2 h}^{s}(r, \theta, \varphi)=-\nabla\left[\lim _{2 h \rightarrow d c} u_{2 h}^{s}(r, \theta, \varphi)\right] \text { for every }(r, \theta, \varphi) \in W
$$

and

$$
\lim _{2 h \rightarrow d c} \mathbf{H}_{2 h}^{s}(r, \theta, \varphi)=-\nabla\left[\lim _{2 h \rightarrow d c} w_{2 h}^{s}(r, \theta, \varphi)\right] \text { for every }(r, \theta, \varphi) \in W .
$$

Consequently, our aim of investigating the stated application, in the low frequency limit, ${ }^{13}$ has been accomplished. Indeed, the scattered electromagnetic fields, in terms of the corresponding harmonic potentials, have been evaluated for the two-sided and non-penetrable, also referred as perfectly electrically conducting, two-hyperboloid. As already mentioned above, the behavior of the strength of the scattered $\left|\mathbf{E}_{2 h}^{s}\right|$ from (69) and the total $\left|\mathbf{E}_{2 h}^{t}\right|$ from (64) with (57) electric field at the cross section $x_{2}$ $=0$ is demonstrated within Figures 5 and 6 for the cases of both a two-hyperboloid (see Figures 5(a) and 6(a)) and the approximation of a double-cone (see Figures 5(b) and 6(b)) of elliptic cross section. Similar behavior is expected for the magnetic field, due to the similarity of Equation (85) with (77), though it is not shown, since in electrostatics the measurable field is the electric one.

The goal of estimating the electric and magnetic fields near corners and edges has been achieved, where, here unlike in the previous application, for the case of a double-cone, either the scattered or the total electric field intensity is significantly diminished at the sharp edge, which is logical if we consider that the source of producing the primary fields is very far from the observed object, hence it redirects accordingly the electric field strength.

\section{CONCLUSIONS}

In this paper, we present a review of ellipsoidal harmonics and an extension of this theory to hyperboloids of one and two sheets. Then, we apply this extension to two boundary value problems of electrostatics. We consider the geometry of certain regions representing edges and corners, whereas either the fields become singular (first application) or vanish (second application), by an elliptic double cone, which stands for the asymptote of the corresponding hyperboloid of two distinct sheets. We then applied the technique in terms of the hyperboloidal harmonics. The electromagnetic fields are explicitly calculated in a purely analytical form for two fundamental cases, the charged metal two-hyperboloid and the metal two-hyperboloid subject to an electric and magnetic plane wave, both of elliptic cross section, where the results are followed by the proper computational elaboration.

In the case where only one of the two sheets of the two-hyperboloid is metallized and charged, the solution for the potential problem becomes more complicated due to the fact that the standard Lamé functions of integer order are excluded. Future elaboration under progress involves the introduction of the Lamé functions of non-integer order. 
This article has endeavored to show that an analytical approach to problems involving ellipsoids or hyperboloids is conceivable and sometimes more straightforward than a numerical solution of relevant integral or differential equations. Once this analysis has been completed, both the credibility and the robustness of numerical implementations are secured.

${ }^{1}$ J. Van Bladel, Singular Electromagnetic Fields and Sources (Oxford University Press, Oxford, 1992).

${ }^{2}$ N. N. Lebedev, I. P. Skalskaya, and Y. S. Uflyand, Worked Problems in Applied Mathematics (Dover Publications, New York, 1965)

${ }^{3}$ A. Passian, S. Koucheckian, S. B. Yakubovich, and A. Thundat, "Properties of index transforms in modeling of nanostructures and plasmonic systems," J. Math. Phys. 51, 023518 (2010).

${ }^{4}$ J. C.-E. Sten and P. K. Koivisto, "Fields and charges near the apex of a hyperbolic cone," Eur. J. Phys. 35, 015019 (2014).

${ }^{5}$ M. Korte and R. Holme, "Regularization of spherical cap harmonics," Geophys. J. Int. 153, 253-262 (2003).

${ }^{6}$ G. Dassios, Ellipsoidal Harmonics: Theory and Applications (Cambridge University Press, Cambridge, 2013).

${ }^{7}$ J. C.-E. Sten, "Ellipsoidal harmonics and their application in electrostatics," J. Electrost. 64, 647-654 (2006).

${ }^{8}$ Y. Shen, D. M. Barnett, and P. M. Pinsky, "Analytic perturbation solution to the capacitance system of a hyperboloidal tip and a rough surface," Appl. Phys. Lett. 92, 134105 (2008).

${ }^{9}$ M. Abramowitz and I. Stegun, Handbook of Mathematical Functions (Dover Publications, New York, 1972).

${ }^{10}$ L. Robin, Fonctions Sphériques de Legendre et Fonctions Sphéroïdales (Gauthier Villars, Paris, 1957).

${ }^{11} \mathrm{~N}$. Engheta, "Electrostatic "fractional' image methods for perfectly conducting wedges and cones," IEEE Trans. Antennas Propag. 44, 1565-1574 (1996).

${ }^{12}$ V. P. Pyati and H. Weil, "Capacitance of biconical antennas in magneto-ionic media; elliptic cone capacitance," J. Res. Natl. Bur. Stand., Sect. D 69D, 291-298 (1965).

${ }^{13}$ G. Dassios and R. E. Kleinman, Low Frequency Scattering (Oxford University Press, Oxford, 2000). 